\title{
A Validated Analytical-Numerical Modelling Strategy to Predict Residual Stresses in Single-Track Laser Deposited IN718
}

\author{
T.R. Walker ${ }^{1}$, C.J. Bennett ${ }^{1}$, T.L. Lee ${ }^{2}$ and A.T. Clare ${ }^{3,4}$
}

${ }^{1}$ Gas Turbine and Transmissions Research Centre, Faculty of Engineering,

University of Nottingham, University Park, Nottingham, NG7 2RD, UK

2ISIS Facility, Rutherford Appleton Laboratory, Harwell Oxford, Chilton, Didcot, OX11 0QX,

$U K$

${ }^{3}$ Advanced Manufacturing Research Group, Faculty of Engineering,

University of Nottingham, University Park, Nottingham, NG7 2RD, UK

${ }^{4}$ Department of Mechanical, Materials and Manufacturing Engineering, Faculty of Science and Engineering, University of Nottingham China, 199 Taikang East Road, University Park,

Ningbo 315100, China

\begin{abstract}
Direct Energy Deposition (DED) is being increasingly used to repair high value components that have been damaged in-service. The uptake of DED and laser cladding operations for repair is inhibited by accurate modelling techniques. Often the repair process required is unique, therefore modelling techniques are necessary to determine the process inputs for the specific application. The DED process subjects the component to high thermal gradients resulting in high magnitude residual stresses and component distortion. Prediction of these parameters would reduce the need for costly experimental trials to quantify the repair strategy. Here, a single-track deposition of IN718, utilising a Nd:YAG laser source and coaxial nozzle, was modelled using a semi analytical-numerical
\end{abstract}


approach. The track profile, temperature fields, melt pool geometry and stress evolutions were simulated for a constant set of process parameters. A corresponding experimental trial was conducted to validate the proposed model, through the use of focus variation microscopy, in-situ temperature measurements, optical micrographs and neutron diffraction measurements. A good correlation between the experimental and numerical data sets were apparent. The track profile was predicted with a maximum error of $1.98 \%$ and $0.43 \%$ for the width and height respectively. The maximum error for the peak temperature and residual stress was $3.1 \%$ and $18 \%$ respectively. Overall, the modelling strategy presented encompasses the key process variables, allowing predictions of the thermal and mechanical effects of the process.

Keywords: Modelling; Direct Energy Deposition; Laser Cladding; Residual Stress; Finite Element; Repair

\section{Introduction}

Direct Energy Deposition (DED) is being increasingly implemented for rapid repair applications. Additive manufacturing techniques such as DED are receiving increased interest in the area of repair due to their improved accuracy, high material utilisation ratio and increased flexibility during manufacture [1]. The development of new repair strategies removes the need to replace high value components, therefore extending the components' service life and increasing economic operation. Typical repair strategies range from applying a thin cladding to the external surface to remedy initial fabrication defects to complete rebuilding of the components structure layer by layer.

The process of DED involves delivering a metallic powder to a metallic substrate with concurrent irradiation of a laser beam [2]. The feedstock material may be delivered in powder, wire or strip form and is often conveyed to the work area in the presence of an inert gas. Various arrangements for this have been reported [2]. During the deposition process, a moving melt pool is generated in the substrate and with controlled material delivery to the molten pool a raised track is formed. Due to the high temperature gradients which occur on cooling of the component, in-situ deformations and residual stresses will be present. In order to assess the effect of the repair process on the components performance it is therefore essential to be able to model these phenomena.

Modelling of laser based DED processes has evolved from simple empirical models to the use of numerical models. The most extensive investigations into the DED process were through experimental testing to derive empirical-statistical models. These early models 
quantified key parameters of the track geometry, but the dependence on experimental data reduces the flexibility of the modelling approach. In recent years, there has been an increase in the number of analytical and numerical models used to represent the DED process [3]. Often an analytical model is used to predict the track geometry as no discretisation of the domain is required. For instance, Picasso et al. [4], predicted the track height by considering the interactions between the powder particles, laser beam and the melt pool. The model was solved iteratively in two coupled sequential steps, however the greatest error in this model was the derivation of the melt pool shape. As the physics of the DED process are interdependent a more representative analytical model was derived by Ahsan et al. [5]. The model derives the overall track profile utilising a fully coupled mass-energy balance. The model was verified through deposition of Ti6Al4V tracks, showing good agreement for both the thermal fields and track dimensions. The analytical approach allows the derivation of the melt pool from an analytical temperature field [6], therefore discretisation of the domain is not required. Numerical models can be used to predict track profile geometries, but there is an increase in complexity and solving times with this approach. For example, a 2D model presented by Ya et al. [7], predicted the track geometry based on a mass-energy balance. The track geometry was determined by analysing the interaction of the powder density and the melt pool, with the final profile being estimated using a parabolic function. Predictions were validated using optical micrographs with good agreement for both the thermal histories and track profile. Pirch et al. [8] utilised a moving meshing technique to simulate the track material by determining its geometry though integration of a particle density function over the melt pool volume. This approach is similar to that of Ahsan et al. [5], however the energy required to melt the powder was not considered. The prediction of the geometry, along with the thermal and residual stress fields were not validated experimentally.

A sequentially coupled thermomechanical analysis solved using the Finite Element (FE) method is the most common approach to predict the thermal and mechanical effects of DED processes [2]. Often the addition of material is modelled using active/inactive or quiet elements, with track profile being represented as a square profile. A study comparing each element strategy, showed that there was less than $0.5 \%$ difference between the derived thermal fields [9]. To date, modelling the development of residual stresses within laser deposited parts has received little attention, due to the high computational complexity and associated cost. Shah et al. [10] and Chew et al. [11] both implemented a thermomechanical model to evaluate the residual stress field of a single-track deposition. The track geometry was represented using square elements for both studies. Validation was conducted through the use of XRD measurements. Shah et al. [10] compared the predictions with experimental measurements for two components of residual stress in the build direction. Both pulsed and continuous laser sources were used in the study, with models showing a good agreement with the experimentally obtained residual stress data. Chew et al. [11] quantified two residual stress components across the mid plane of the substrate. Again, good agreement with the experimental residual stress profiles was presented, with the longitudinal stress 
profile following the same trend as a traditional single weld pass. The in-situ temperature histories agree well with the FE models for both authors. A recent study by Pirch et al. [12] utilised a three-dimensional time-dependant FE model which predicts the track profile and the subsequent time-evolution of temperature and stresses. An in-house simulation tool was developed to simulate the process. Experimental validation was conducted for the track profile only, showing a good agreement between the data sets. The sequentially coupled thermomechanical analysis was extended by Mukherjee et al. [13] to include the fluid flow of the melt pool in the thermal analysis by solving the mass, energy and momentum equations in a 3D discretised domain. The domain consisted of a square substrate with a rectangular geometry used to represent the deposited wall structure. The predicted thermal histories agreed well with the experimental data with a maximum error of $2.1 \%$. Validation of the mechanical model was conducted using independent experimental results through comparison of the predicted longitudinal and through thickness stress components with XRD data. Despite the inclusion of a more accurate representation of the weld pool, a maximum error of $62 \%$ and $40 \%$ for each of the stress components was observed respectively, with the predicted data set falling outside the confidence limits for the experimental data. It should be noted that a full triaxial stress comparison was not presented. The use of the FE method to predict the mechanical effects of the DED process is computationally expensive, therefore a method devised by Liang et al. [14] aimed to reduce the analysis time by using a modified inherent strain method. The modified method includes the accumulated elastic strain as part of the inherent strain, as this component causes distortion in the remaining build. To ensure the modelling approach was practical, a small scale, detailed model was used to determine the inherent strain. The extracted inherent strain was then applied to single wall structures of varying layers to predict residual deformation. An experimental validation case was utilised by comparing the measured and predicted distortion from both the small-scale model and a full-scale process model. Although the proposed strategy reduces computational time by up to 80 times for specific cases and provided a good estimate for the total deformation (maximum error of 6.6\%), the accuracy of the modelling strategy was not evaluated for predicting residual stress. It should also be noted that a rectangular geometry was used to represent the deposited track in all of the simulations.

The aim of this study is to validate, through experimental investigation, a semi analyticalnumerical approach, which allows the prediction of the thermal and mechanical effects of the process. The modelling methods adopted in this study provide superior fidelity of the deposition process through inclusion of a predicted track geometry in a sequentially coupled thermomechanical FE analysis. Through implementing a more realistic representation of the deposition geometry, accurate predictions of the substrate deformation and the resultant tri-axial residual stress profiles at three horizontal locations are presented. 


\section{Methodology}

\subsection{Computational Model}

A semi analytical-numerical approach was used to model the process in this study. A flowchart of the modelling strategy is presented in Figure 1. An iterative analytical model was implemented to predict the track profile as presented by Ahsan et al. [5]. The melt pool profile was derived from an analytical temperature field calculated using the Cline and Anthony equation [6] for a moving Gaussian heat source. The equation gives the temperature field at the substrate, based on a non-dimensional distribution function. The associated power losses are accounted for in the analytical model by including the evaporation power loss $\left(P_{\text {evap }}\right)[15]$ at the melt pool and the power required to sustain mass addition $\left(P_{m}\right)$ [5]. The powder distribution on the substrate surface was assumed to be Gaussian [16] with the Gaussian feed radius being calculated as $2.0 \mathrm{~mm}$ for this work [17]. The material properties used for the analytical model are outlined in Table 1 . The values were assumed to be temperature independent and were defined at a temperature halfway between the melting $\left(1260^{\circ} \mathrm{C}\right)$ and ambient temperature $\left(20^{\circ} \mathrm{C}\right)$ of the substrate [5]. The calculated track profile was then used in a sequentially coupled thermomechanical FE model to predict the thermal and mechanical induced effects of the process. The numerical models assume that the formation of residual stress is driven predominately through thermal effects. Therefore, mass transfer, fluid flow and phase transformations were not included in the analyses [18].

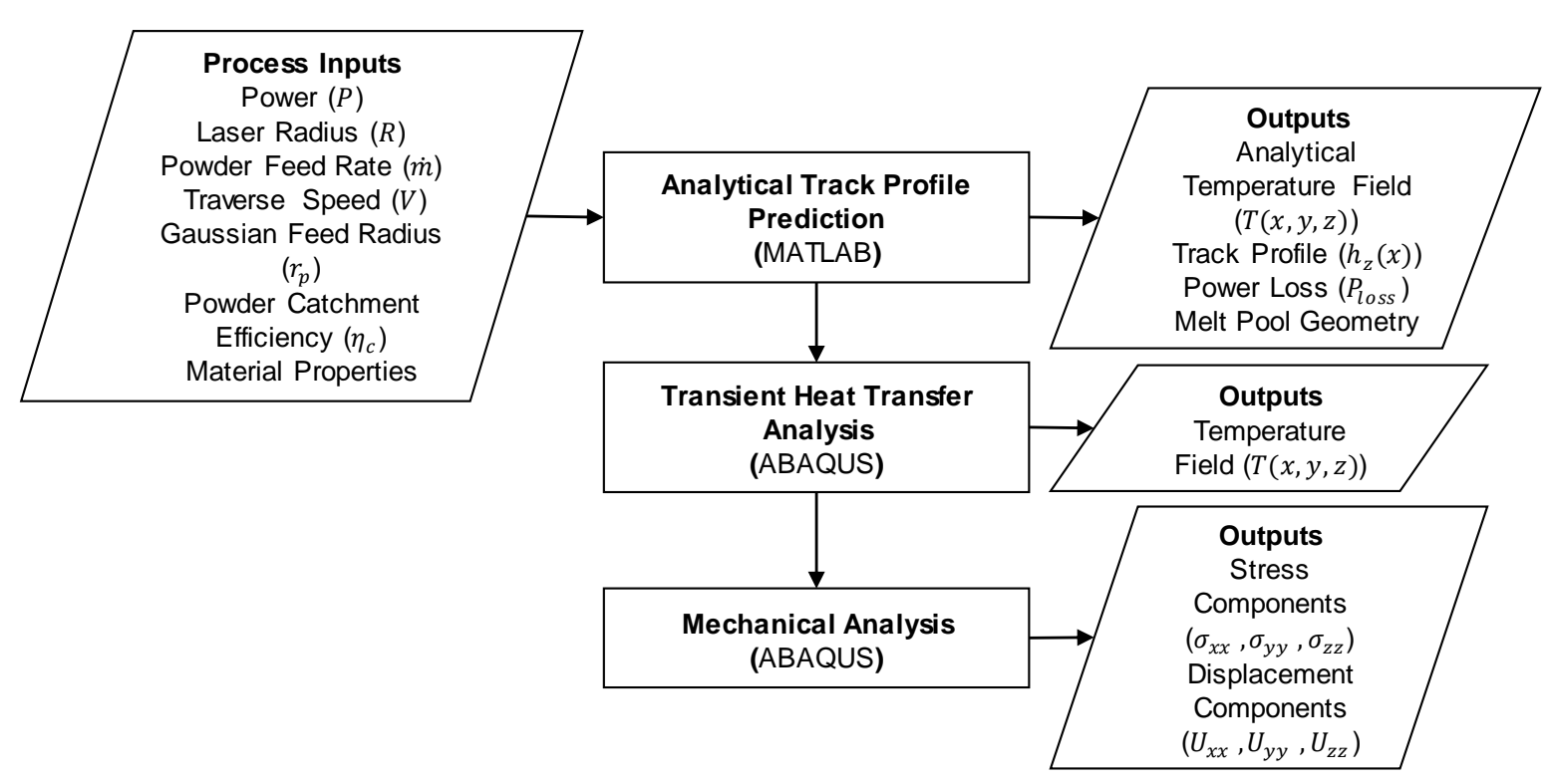

Figure $1 \mathrm{~A}$ flowchart showing the required inputs for the model and the associated outputs

at each stage of the modelling process. 
Table 1 Thermal properties for IN718 used for the analytical portion of the model.

\begin{tabular}{llc}
\hline Density $\left(\mathrm{kg} / \mathrm{m}^{3}\right)$ & {$[19]$} & 7993 \\
Thermal Conductivity $\left(\mathrm{W} / \mathrm{m}^{2} \mathrm{~K}\right)$ & {$[19]$} & 21 \\
Specific Heat Capacity $(\mathrm{J} / \mathrm{KgK})$ & {$[19]$} & 536 \\
Latent Heat of Melting $(\mathrm{J} / \mathrm{Kg})$ & {$[20]$} & 210 \\
Latent Heat of Vaporization $(\mathrm{kJ} / \mathrm{Kg})$ & {$[21]$} & 6400 \\
Solidus Temperature $(\mathrm{K})$ & {$[20]$} & 1533 \\
Liquidus Temperature $(\mathrm{K})$ & {$[20]$} & 1609 \\
Laser Absorptivity & {$[22]$} & 0.3 \\
\hline
\end{tabular}

\subsubsection{Powder Catchment Efficiency}

The DED method in the study utilises a blown powder feed. Therefore, the catchment efficiency had to be accounted for in the analytical model. The model presented by Ahsan et al. [5] assumed that all the powder bound by the melt pool is assimilated, therefore the catchment efficiency was equal to:

$$
\eta_{c_{1}}=\frac{\int_{-\frac{W}{2}}^{\frac{W}{2}} \int_{y_{r}}^{y_{f}} q_{p f} d y d x}{\int_{-\infty}^{\infty} \int_{-\infty}^{\infty} q_{p f} d y d x}
$$

A more realistic approximation of the catchment efficiency was derived by Partes [23]. It should be noted that for consistency the integration limits have been modified from Partes [23] to reflect the melt pool geometry derived by Ahsan et al. [5]:

$$
\begin{gathered}
\eta_{c_{2}}=\eta_{1}+\eta_{2} \\
\eta_{1}=\int_{-\frac{W}{2}}^{\frac{W}{2}} \int_{y_{r}}^{y_{f}} S(x, y) d y d x
\end{gathered}
$$




$$
\begin{gathered}
\eta_{2}=\int_{-\infty}^{0} \int_{0}^{\sqrt{\left(\frac{w}{2}\right)^{2}-\frac{X_{\min }^{2}}{4}}} S(x, y) d y d x-\left[\int_{y_{r}}^{0} \int_{0}^{\left(\frac{w}{2}\right) \sqrt{1-\frac{x^{2}}{y_{r}^{2}}}} S(x, y) d y d x\right. \\
\left.-\int_{\frac{y_{r} X_{\min }}{W}}^{0} \int_{\left(\frac{w}{2}\right)^{2}-\frac{x_{\min }^{2}}{4}}^{\frac{\left(\frac{w}{y_{r}^{2}}\right.}{1-\frac{x^{2}}{2}}} S(x, y) d y d x\right] \\
S(x, y)=\frac{1}{2 \pi \sigma_{x} \sigma_{y}} \exp \left[-\frac{1}{2}\left(\frac{x}{\sigma_{x}^{2}}+\frac{y}{\sigma_{y}^{2}}\right)\right] \\
X_{\text {min }}=\frac{\pi D_{\text {particle }} \rho_{\text {powder }} C_{p}\left(T_{m}-T_{\text {amb }}\right)(2 R)^{2} v_{\text {powder }}}{6 \alpha_{\text {powder }} P}
\end{gathered}
$$

where $\sigma_{x}$ and $\sigma_{y}$ are geometrical constants as detailed in [23], $D_{\text {particle }}$ is the mean powder diameter, $\rho_{\text {powder }}$ and $\alpha_{\text {particle }}$ are the density and absorptivity of the powder assumed to equal that of the substrate material. The velocity of the powder ( $\left.v_{\text {powder }}\right)$ was given by [17]:

$$
v_{p o w d e r}=\frac{4 \dot{V}}{\pi\left(d_{0}^{2}-d_{i}^{2}\right)}
$$

where $\dot{V}$ is the volumetric flow rate of the carrier gas, $d_{0}$ is the outer diameter of the coaxial nozzle and $d_{i}$ is the inner diameter. A modified catchment efficiency based on the two methods was given by:

$$
\eta_{c}=\frac{\eta_{c_{2}}}{\eta_{c_{1}}}
$$

\subsubsection{Numerical Modelling}

Computational Welding Mechanics (CWM) was used to model the process due to the similarity with traditional welding methods [24]. As the deformations have negligible effect on the thermal fields a sequentially coupled thermomechanical FE analysis was implemented. This technique involved solving the temperature field independently from the mechanical analysis. The thermal histories were then superimposed as thermal strain boundary conditions to solve for displacement and residual stress. 
Material properties selected for the numerical model were temperature dependent as shown in Figure 2. Poisson's ratio, was assumed to be independent of temperature equating 0.33 [19]. The material properties were applied to the substrate and track geometry. It should be noted that although there will be metallurgical difference in the grain size, microstructure and with dissolution and recrystallisation occurring in the deposited track and in the vicinity of the melt pool, the same material properties were applied to both regions. It was found by Deshpande et al. [25] that modifications to the material properties to match that of the melt pool region had negligible effect on the residual stress profiles. All FE simulations in this work were conducted using the commercial FE software ABAQUS, to allow comparisons to be drawn between predicted and experimental residual stress profiles.

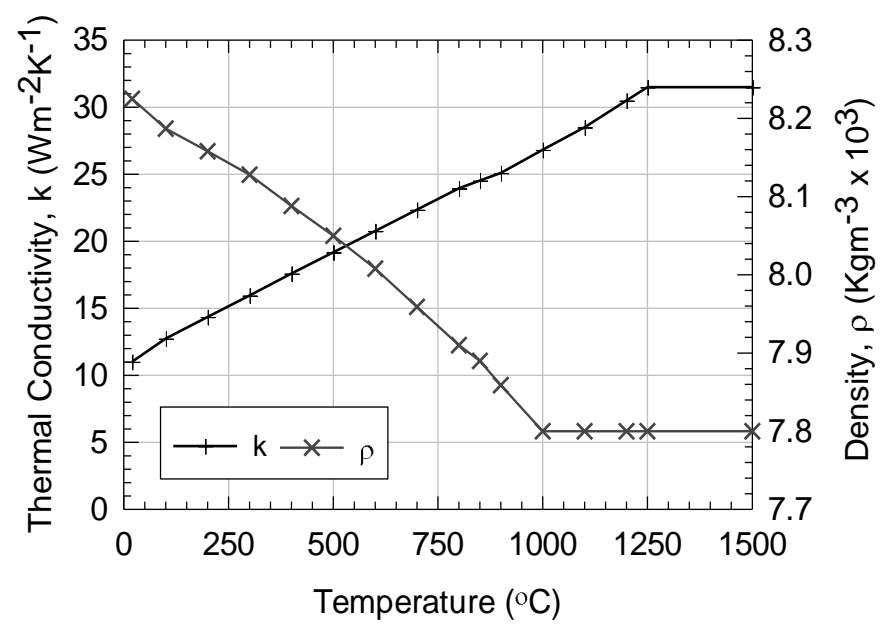

(a)

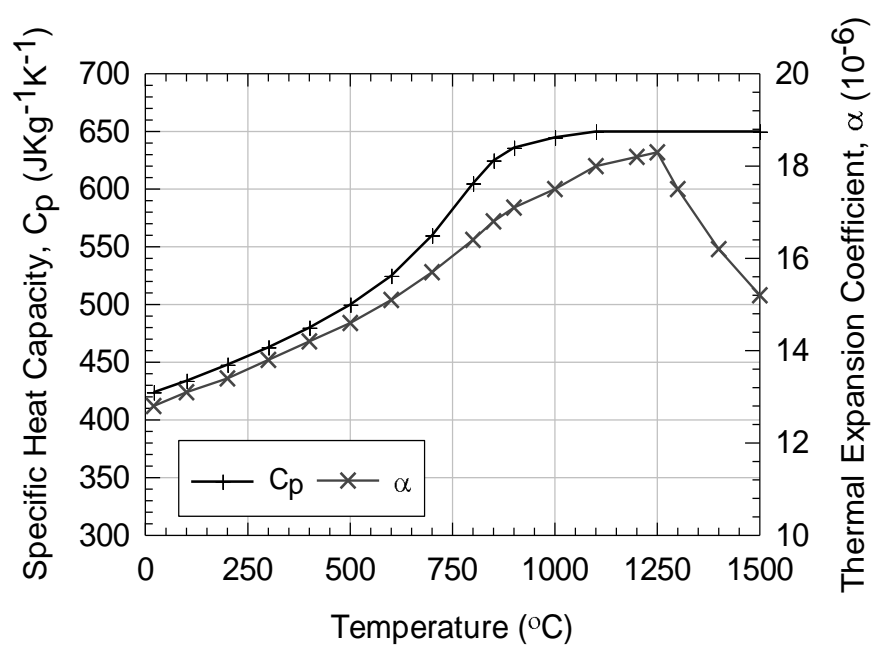

(b) 


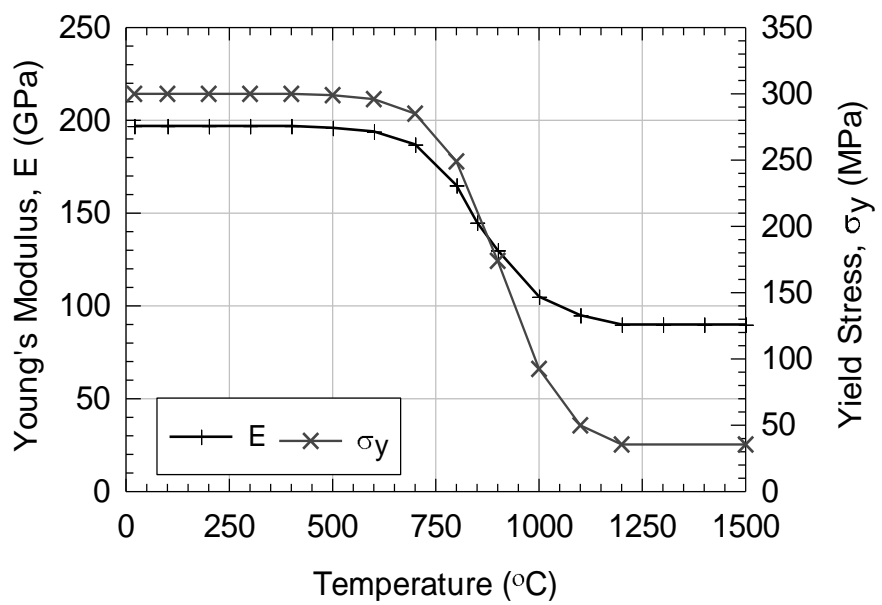

(c)

Figure 2 Temperature dependant thermomechanical properties of IN718 used for all FE models; (a) Thermal conductivity, $k$, and density, $\rho$; (b) Specific heat capacity, $C_{p}$, and thermal expansion coefficient, $\alpha$; (c) Young's modulus, $E$, and yield stress, $\sigma_{y}[19]$.

\subsubsection{FE Mesh}

A 3D FE model was utilised with only half of the geometry modelled due to symmetry along the deposition track. The nodal positions at the interface between the track and substrate were identical. The nodes in this region were joined using a tie constraint. Square elements were used throughout the mesh to allow post processing of the data to reflect the method used for the ND measurements.

A mesh sensitivity study was conducted for the thermal model. For each iteration of the convergence study the element volume was reduced by half until convergence was achieved. The starting element volume in the vicinity of the track was $1 \times 2 \times 1 \mathrm{~mm}^{3}$. Through the use of mesh biasing the element volume was increased from $2 \times 2 \times 1 \mathrm{~mm}^{3}$ to $8 \times 2 \times 1 \mathrm{~mm}^{3}$ at the extremity of the substrate width. A constant element size was utilised through the thickness of the substrate until $4 \mathrm{~mm}$ through the depth. For each element volume, the integration time step was selected so that the FE heat source model moved one element length per each integration time step. To determine if the reduction in element volume for each iteration was beneficial, the coefficient of determination $\left(R^{2}\right)$, calculated for a thermal history and the fusion zone profile, for the previous and current element size was utilised. The nodal coordinate used to extract the thermal history from the FE model was located at the mid length of the substrate, at a nodal position $3 \mathrm{~mm}$ from the track centreline, on the top surface of the substrate. The geometry of the fusion zone was extracted at the substrate mid length at a time period where the fusion zone was at its maximum width. To be consistent with the analytical approach used in this study, mesh independence was achieved when the coefficient of determination for the thermal history and the profile of the fusion 
zone were greater than 0.995 [26]. Upon convergence being met, the previous element volume was selected and used for the mesh design. The results of the mesh study are presented in Figure 3.

Transverse Direction Element Length (mm)

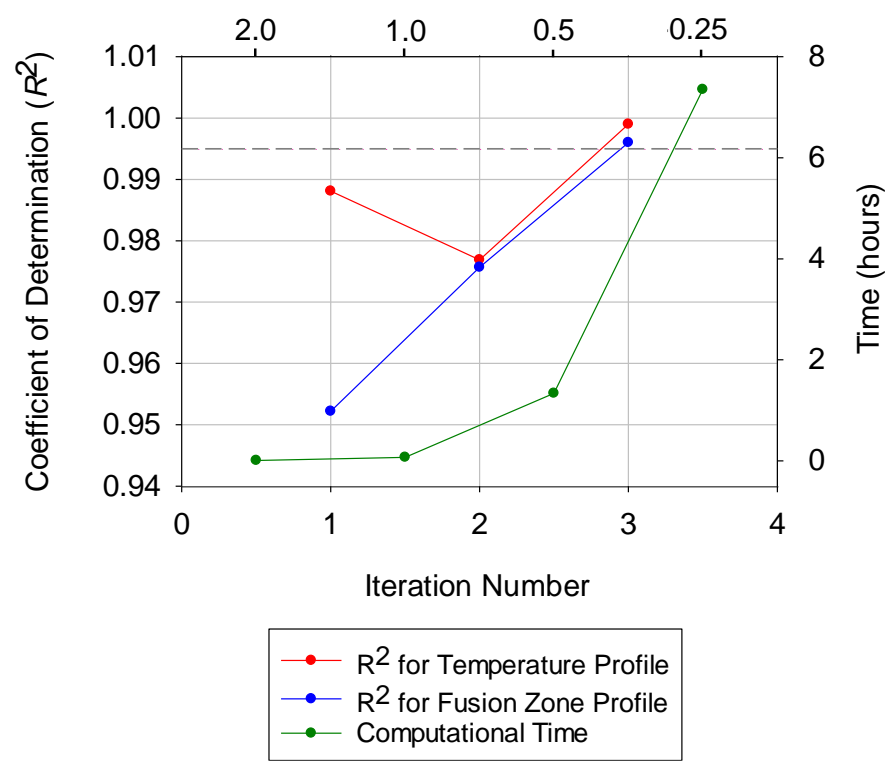

Figure 3 Mesh sensitivity study results showing the calculated convergence parameters for each mesh iteration. Computational time presented for each of the four element sizes used in the study. The minimum value required for mesh convergence shown by the dotted grey line.

From the results in Figure 3 the third iteration satisfies the convergence limits of the mesh sensitivity study. A reduction in accuracy is observed for the second iteration, arising due to the finer element size being able to represent the heat flux distribution of the FE heat source with greater accuracy. As expected, the computational time increases exponentially through reduction of the element volume. The resultant mesh from the study is presented in Figure 4. The element volume directly below the track was $0.25 \times 0.5 \times 0.25 \mathrm{~mm}^{3}$ increasing from $0.5 \times 0.5 \times 0.25 \mathrm{~mm}^{3}$ to $2 \times 0.5 \times 0.25 \mathrm{~mm}^{3}$ at the extremity of the plate. The mesh design in Figure 4 was also implemented for the mechanical model. As the thermal solution was independent of the mesh, an accurate prediction of the transient temperature field could be achieved which is required to predict the residual stress fields with accuracy [14]. The increase in element size far field from the vicinity of the track was also valid because only the stiffness of the material needs to be represented after the melt pool zone. DC3D8 linear brick elements and C3D8R linear brick elements were used for the thermal and mechanical analysis respectively. Linear elements were used as there are no significant advantages in the results produced by higher order elements in welding simulations [27]. The total number of elements and nodes were 196,200 and 213,294 respectively. 


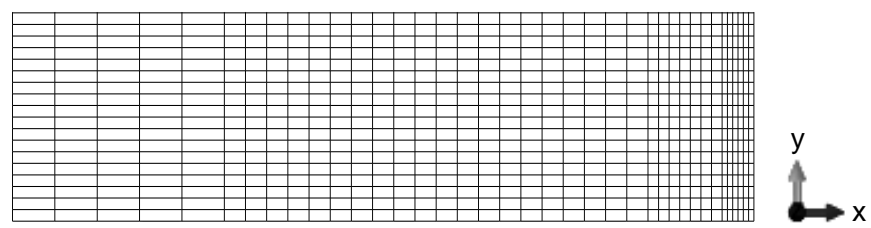

(a)

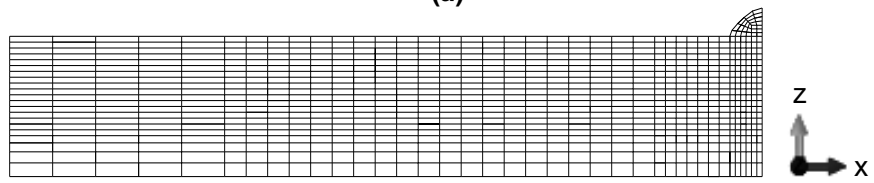

(b)

Figure 4 A Section of the FE mesh used for the thermomechanical models; (a) Top surface view; (b) Cross sectional view perpendicular to the track.

\subsubsection{Thermal Analysis}

The governing transient heat transfer equation for a stationary medium has the following form [28]:

$$
\rho C_{p} \frac{\partial T}{\partial t}(x, y, z, t)=-\nabla \cdot \vec{q}(x, y, z, t)+Q(x, y, z, t)
$$

where $T$ is the current temperature, $Q$ is the internal heat generation rate, $\nabla$ is the spatial gradient operator and $\vec{q}$ is the heat flux vector equalling the non-linear Fourier heat flux constitutive equation [29]:

$$
\vec{q}=-k \nabla T
$$

Thermal effects, due to melting/solidification of the melt pool and deposition material, were included in the model by taking into consideration the latent heat of melting (Table 1) for the respective solidus and liquidus temperatures $\left(1260^{\circ} \mathrm{C}\right.$ and $\left.1336^{\circ} \mathrm{C}\right)[20]$. For consistency between the analytical and numerical model, the circular disc heat source proposed by Pavelic et al. [30] was used:

$$
Q(x, y, z, t)=\frac{\alpha P-\left(P_{m}+P_{\text {evap }}\right)}{\pi r_{0}^{2}} \exp \left[-\frac{\left(x^{2}+(y-V t)^{2}\right)^{2}}{r_{0}^{2}}\right]
$$

where $r_{0}$ is the Gaussian radius equating $1.5 \mathrm{~mm}$ based on the experimental track width. The heat source model was implemented using the user-subroutine DFLUX in ABAQUS. The FE domain was solved using the full Newton-Raphson integration scheme. An integration time 
period equal to moving one element length per time step was adopted for the heating steps of the analysis only. The integration time was set to automatic for the cooling phase. The active/inactive element approach was used to model the deposition of material, with all elements being deactivated at the start of the analysis. To ensure that the energy balance inside the melt pool was stable, the elements were reactivated offset from the centre of the melt pool. A total width of two elements were reactivated at each time step at the melting temperature of the material. The deposition strategy implemented is outlined in Figure 4.

Convective and radiative losses were accounted for using the Newton and StefanBoltzmann laws respectively. The convective heat transfer coefficient and emissivity were set to $25 \mathrm{~W} / \mathrm{m}^{2} \mathrm{~K}$ and 0.8 respectively [25]. As varying portions of the substrate surface were obscured by track material during the analysis, the convection and radiation losses were defined only on the free surfaces visible to the external surroundings. A convective boundary condition was also applied to the bottom surface of the substrate, to model the heat loss between the CNC table and the substrate due to the heat sink effect. Often a higher value, based on trial and error, is required for the heat transfer coefficient to model the higher rate of energy exchange between the substrate and the bed [31]. A value of $1200 \mathrm{~W} / \mathrm{m}^{2} \mathrm{~K}$ was used in this work. The sink temperature for all the defined losses and the initial substrate temperature were set to $20^{\circ} \mathrm{C}$.

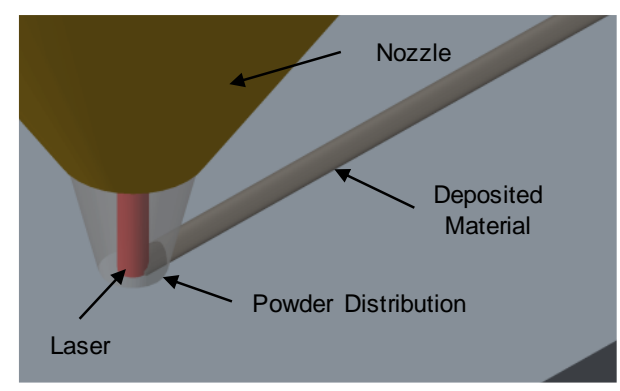

(a)

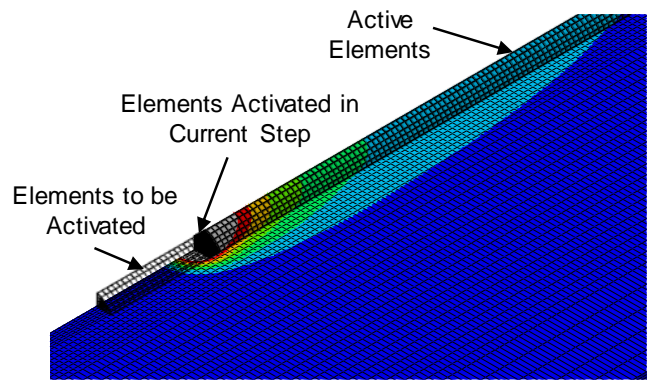

(b)

Figure 5 (a) Schematic of the deposition process; (b) Adopted FE modelling strategy for element activation to simulate the deposition process.

\subsubsection{Mechanical Analysis}

The FE mesh utilised in the thermal model was also used for the mechanical analysis so the nodal coordinates were identical, thus allowing the models to be coupled. As there are no solid state phase transformations in IN718 from room temperature to its melting point [32], the total strain can be divided into three components [29]:

$$
\varepsilon_{T}=\varepsilon_{e}+\varepsilon_{p}+\varepsilon_{t h}
$$


where the components of strain are elastic $\left(\varepsilon_{e}\right)$, plastic $\left(\varepsilon_{p}\right)$ and thermal respectively $\left(\varepsilon_{t h}\right)$. The elastic strain component was modelled as isotropic using Hooke's law and the plastic strain was determined using the von Mises yield criterion. An isotropic hardening model, with a hardening coefficient of $0.01 E$ was used to represent the plastic behaviour [19]; this model is acceptable due to the limited amount of cyclic loading, as no reheating is present in a single-track deposition. The thermal strain was evaluated using the thermal history of the heating and cooling phase in conjunction with the thermal expansion coefficient. As the feedstock material and substrate enters a molten state during deposition, the associated volume change due to the melting/solidification of the material needs to be included. This phenomenon was accounted for in the mechanical model by utilising the annealing function in ABAQUS. This function allows the effects of melting and solidification in metals to be accounted for during high temperature material processing [33]. This function causes the accumulated plastic strain, due to work hardening, to be reset to its initial condition if the melting temperature of the material is exceeded. If the temperature falls below the melting temperature, then plastic strain can be accumulated again. The annealing temperature was set to the solidus temperature for IN718 $\left(1260^{\circ} \mathrm{C}\right)$. It should be noted that volume change due to phase transformations upon solidification were neglected. As no fixed clamps were used in the experimental set up, mechanical boundary conditions were applied in the model to only restrict rigid body motion. Material addition was included in the mechanical analysis using the same deposition strategy as in the thermal analysis.

\subsection{Experimental Methodology}

The deposition system used in this work (Figure 6) was a YC50 cladding head, with a $2 \mathrm{~kW}$ Ytterbium doped, continuous wave, fibre laser (IPH Photonics) operating at a wave length of $1070 \mathrm{~nm}$. A four-feed coaxial nozzle was used to deliver powder using argon carrier gas from a Praxair (Model 1264) powder feeder. Gas atomised IN718 powder with a size ranging from $45 \mu \mathrm{m}$ to $15 \mu \mathrm{m}$ was used in the experiment. The laser beam was operated at a standoff distance of $12 \mathrm{~mm}$, which produced a $2.6 \mathrm{~mm}$ spot diameter. The powder feed and argon gas were set to $4 \mathrm{rev} / \mathrm{min}$ and $10 \mathrm{~L} / \mathrm{min}$ respectively. In order to obtain a mass flow rate, calibration of the powder feed was conducted by measuring the mass of powder over a duration of one minute. 


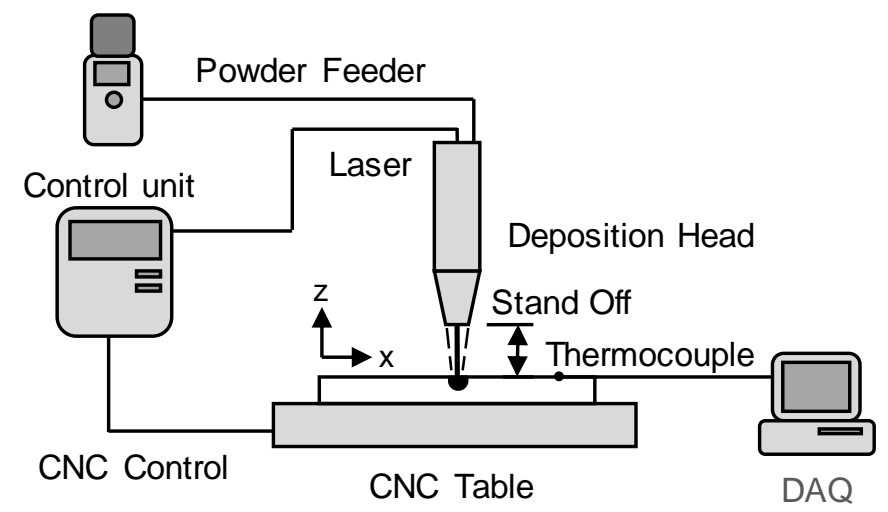

Figure 6 Schematic of the experimental apparatus.

A sand-blasted IN718 plate $(120 \times 70 \times 6 \mathrm{~mm})$, supplied by Haynes International (Manchester, UK.), in the solution heat treated state was used for the substrate. The chemical composition of the material is presented in Table 2. Two identical samples, denoted sample $A$ and sample B, were manufactured with a schematic of the experimental process shown in Figure 7. Sample A was used for in-situ temperature measurements, metallurgical examination and for Neutron Diffraction (ND) measurements. Sample B allowed production of the strain-free reference $\left(d^{0}\right)$ sample. Four bolts, acting as simple supports, were positioned along the width and length to restrict the plate's motion during the deposition process.

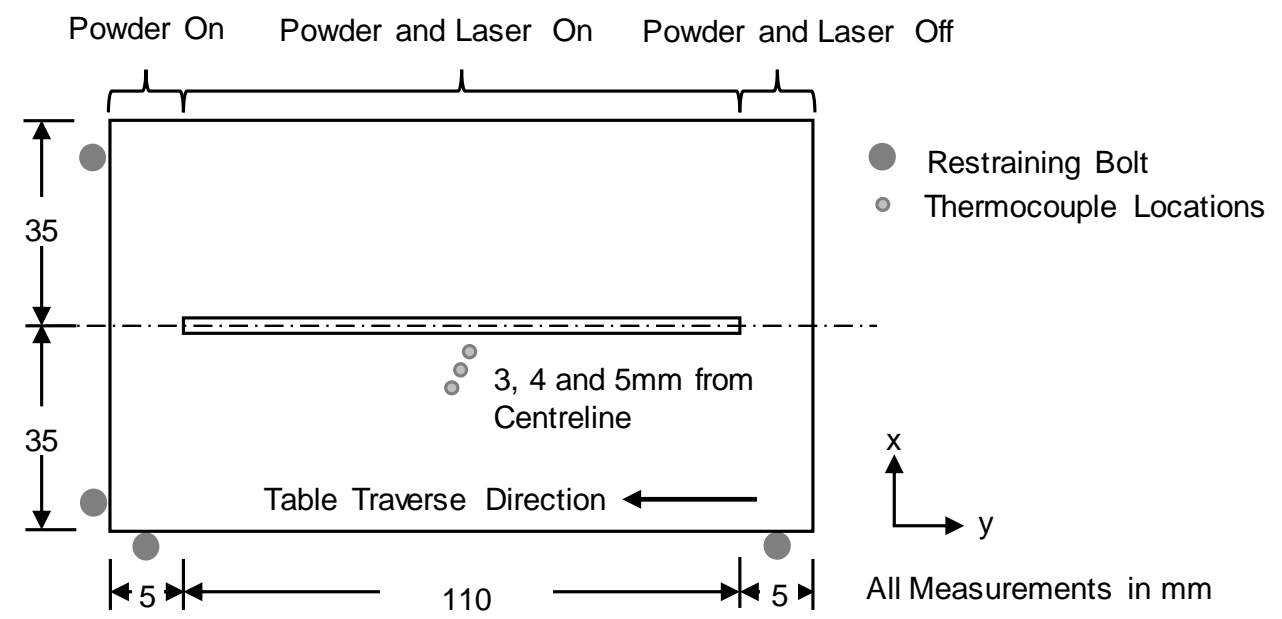

Figure 7 Schematic of the experimental set up showing the deposition procedure, restraining locations and thermocouple locations.

Table 2: Chemical composition of IN718 in wt\%.

\begin{tabular}{cccccccccccc}
\hline $\mathbf{N i}$ & Co & Fe & Cr & Co+Ta & Mo & Mn & Si & Ti & Al & C & B \\
\hline 52 & 1 & 19 & 18 & 5 & 3 & 0.35 & 0.35 & 0.9 & 0.5 & 0.05 & 0.004 \\
\hline
\end{tabular}


The process parameters were selected based on previous work using this apparatus [34] and are presented in Table 3. Three sequential steps were used in the experiment. For the first $5 \mathrm{~mm}$ of the traverse, powder was delivered to the substrate with no heating. After this point, the laser was switched on to begin formation of the track. After a distance of $115 \mathrm{~mm}$ both the laser and powder feed were switched off. The sample was then cooled in air until it was at room temperature. In-situ temperature measurements were conducted utilising Ktype thermocouples. Each junction was spot welded in the exposed condition at the mid length of the substrate. The thermocouples were positioned at three different locations from the centreline (Figure 7). The distance to the centreline was minimal to capture the localised heating affects. Data was captured using a National Instrument SCXI-1000 chassis in conjunction with a SCXI-1102B voltage input module. The data was logged using LabVIEW for processing and analysis.

Table 3 Process parameters used for the experimental deposition.

\begin{tabular}{lc}
\hline Laser Power $(\mathrm{W})$ & 1800 \\
Traverse Velocity $(\mathrm{mm} / \mathrm{min})$ & 400 \\
Powder Feed Rate $(\mathrm{g} / \mathrm{min})$ & 26 \\
Traverse Distance $(\mathrm{mm})$ & 120 \\
\hline
\end{tabular}

\subsubsection{Track Profile and Metallographic Analysis}

To validate the melt pool geometry metallographic analysis was conducted on a specimen $(20 \times 10 \times 6 \mathrm{~mm})$ removed from sample A. The cutting location is presented in Figure 8.

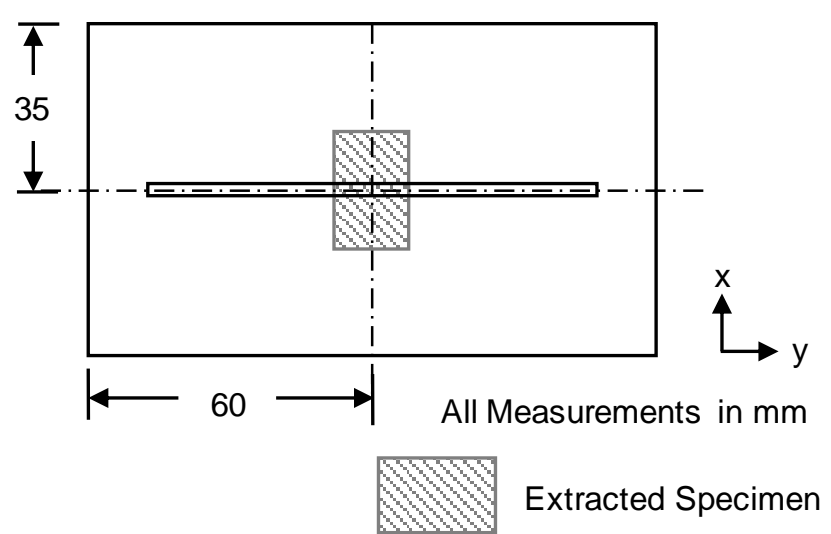

Figure 8 Location of extracted specimen from sample a used for metallographic analysis. 
The specimen was removed by waterjet cutting and mounted in conductive resin using a 30 $\mathrm{mm}$ press. Grinding of the specimen surface using $\mathrm{SiC}$ abrasive paper to 1200 grit size was completed, followed by polishing using a 6 and $1 \mu \mathrm{m}$ diamond wheel. The specimen was chemically etched using Marble's Reagent $\left(10 \mathrm{~g} \mathrm{CuSO}_{4} 50 \mathrm{ml} \mathrm{HCL}\right.$ and $\left.50 \mathrm{ml} \mathrm{H}_{2} \mathrm{O}\right)$. The etchant was applied using a swabbing technique and was left on the surface of the material for no longer than 10 seconds. The fusion zone was inspected using a digital optical microscope. The track profile and the camber distortion of the substrate were measured through focus variation microscopy.

\subsubsection{Neutron Diffraction Measurements}

ND measurements were conducted using the ENGIN-X facility at the ISIS neutron source at the Rutherford Appleton Laboratory, UK [35]. During the experiment, two strain components were collected simultaneously with the use of two directly opposing detector banks at $2 \theta= \pm 90^{\circ}$. Through rotation of the sample a full tri-axial stress analysis was conducted. The set-up of the sample in the beamline is shown in Figure 9. In both orientations, three line-scans were adopted at three locations across the length of the sample (Figure 10).
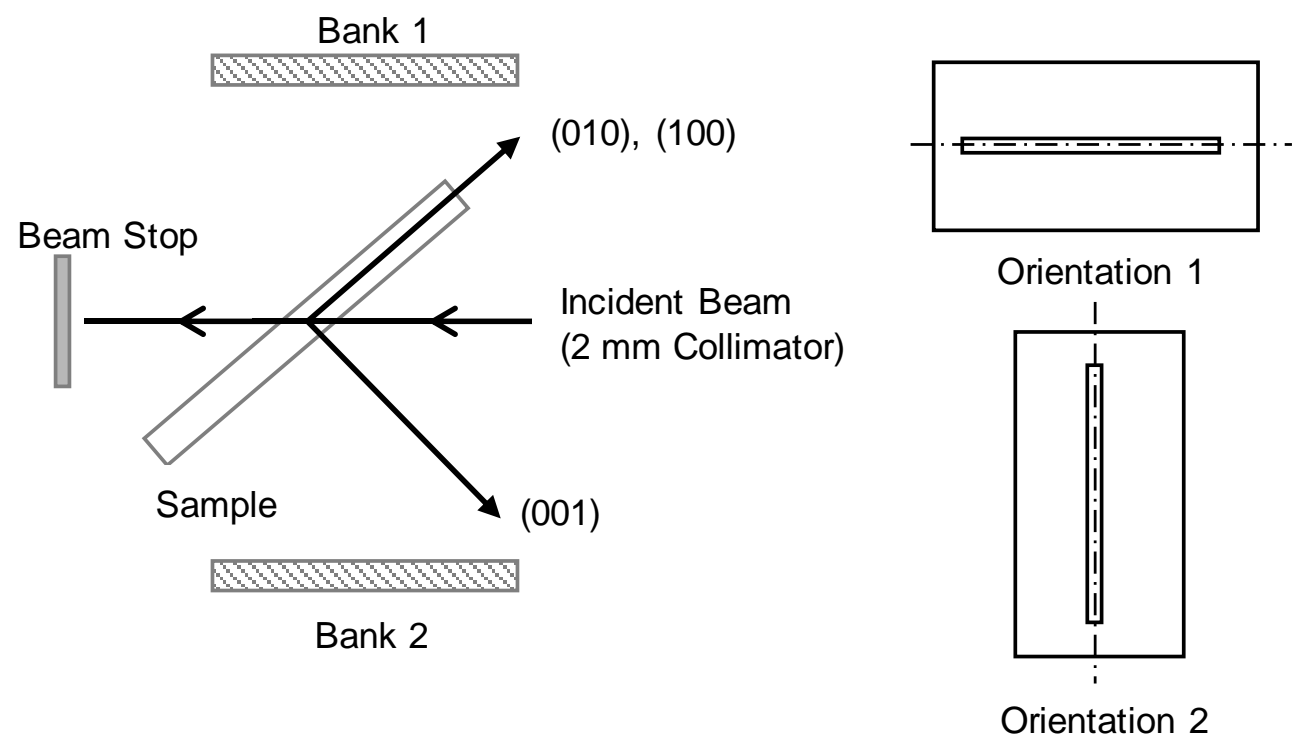

Figure 9 Schematic of the ENGIN-X beamline setup used to obtain ND measurements showing the two plate orientations used, the incident and diffracted beam and the measured strain directions.

All measurements were conducted to the right of the sample centreline due to the geometrical symmetry, at $2 \mathrm{~mm}$ below the surface of the substrate. A gauge volume of $2 \times 2$ 
x $2 \mathrm{~mm}^{3}$ was used for all measurements allowing for a good compromise between accuracy of the measurements and the spatial resolution at which they were conducted.

To obtain the strain-free lattice spacing, two $d^{0}$ specimens were required because of the spatial resolution of each measurement. These specimens were removed from sample $B$ using electrical discharge machining (EDM) at the location highlighted in Figure 10. To relieve the internal strain, EDM was used to cut the $d^{0}$ specimens into a comb specimen [36] as presented in Figure 11. An increased spatial resolution (smaller spacing between ND measurement positions) was used in the proximity of the deposited track to quantify the sharp gradient in residual stress. The spatial resolution was reduced in regions away from the deposited track as the stress gradients were expected to be lower. For each measurement, the incident beam was focused at the centre of each tooth on the comb specimen at a distance of $2 \mathrm{~mm}$ from the top surface. The same spatial resolution used to acquire the strain-free lattice spacing was used for the measurements in sample A.

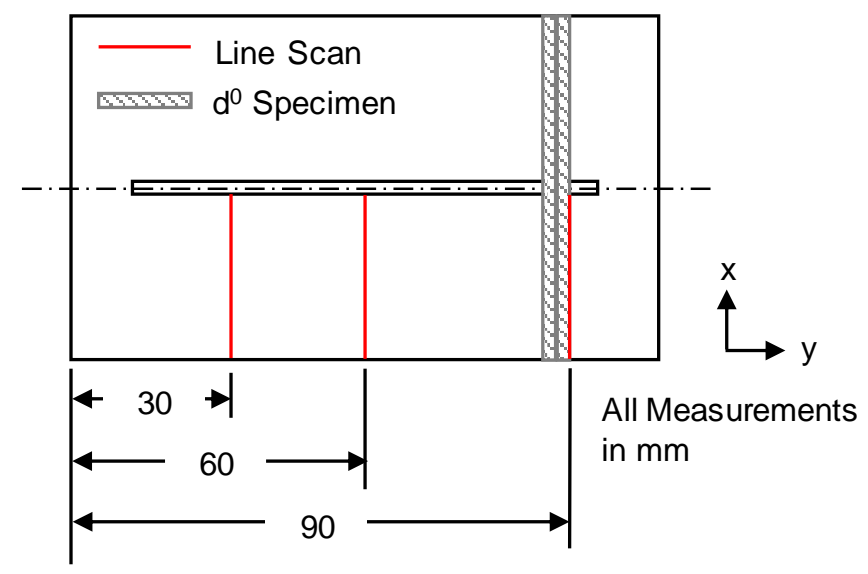

Figure 10 Schematic of sample B showing the locations of the horizontal line scans (red lines) and the location where the $d^{0}$ specimens were extracted (hatched boxes).
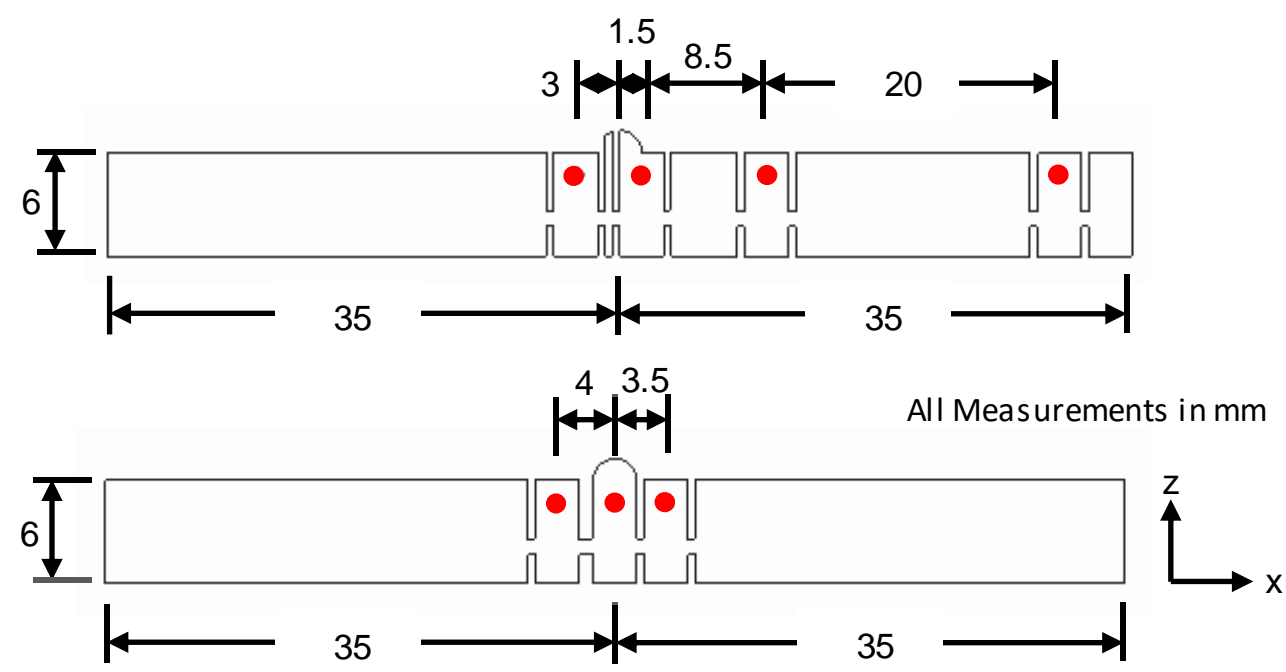
The Rietveld method was used to determine the lattice spacing by minimising the difference between the experimental and calculated diffraction pattern. This method was conducted using the General Structure Analysis System (GSAS) designed to post process diffraction data obtained from using X-rays or neutrons. The elastic lattice strain was calculated as follows:

$$
\varepsilon=\frac{d-d^{0}}{d^{0}}
$$

where $d^{0}$ is the strain free lattice spacing and $d$ is the lattice spacing of interest. As the lattice strain was calculated along each principle direction of the samples, the macroscopic stress components in the $i$ direction were obtained from Hooke's law utilising the following formulae:

$$
\sigma_{i j}=\frac{E}{1+v}\left[\varepsilon_{i j}+\frac{v}{1-2 v}\left(\varepsilon_{11}+\varepsilon_{22}+\varepsilon_{33}\right)\right]
$$

where the numerical subscripts denote the direction in the sample, $E$ is the Young's modulus and $v$ is the Poisson's ratio, having values of $209 \mathrm{GPa}$ and 0.29 respectively [37].

\section{Results}

\subsection{Track Profile Prediction}

A total of 26 cross sectional profiles were extracted from the focus variation microscopy images. From this data, a steady-state region was identified (Figure 12) as the location where the track height/width did not vary by $\pm 5 \%$ from one profile to the next. An average profile was determined from this region. Comparisons of the analytical and experimental track profiles are presented in Figure 13. Three track profiles were calculated utilising the catchment efficiencies $\eta_{c}, \eta_{c_{1}}$ and $\eta_{c_{2}}$. These values were derived as $0.51,0.80$ and 0.41 respectively. It should be noted that a converged solution was not obtained for $\eta_{c_{1}}$ as no stable melt pool could be achieved. Utilising the analytical catchment efficiency presented by Partes [23] gives rise to an underestimate in the track height of $16.4 \%$. However, the track width and shape is represented well by the analytical model. When taking into consideration the combined catchment efficiency $\left(\eta_{c}\right)$ the track width and height are predicted with an error of $1.98 \%$ and $0.43 \%$ respectively. Overall, the profile shape is in good agreement with 
the experimental data. The coefficient of determination $\left(R^{2}\right)$ was derived as 0.982 , with the greatest difference arising at the position of maximum width.

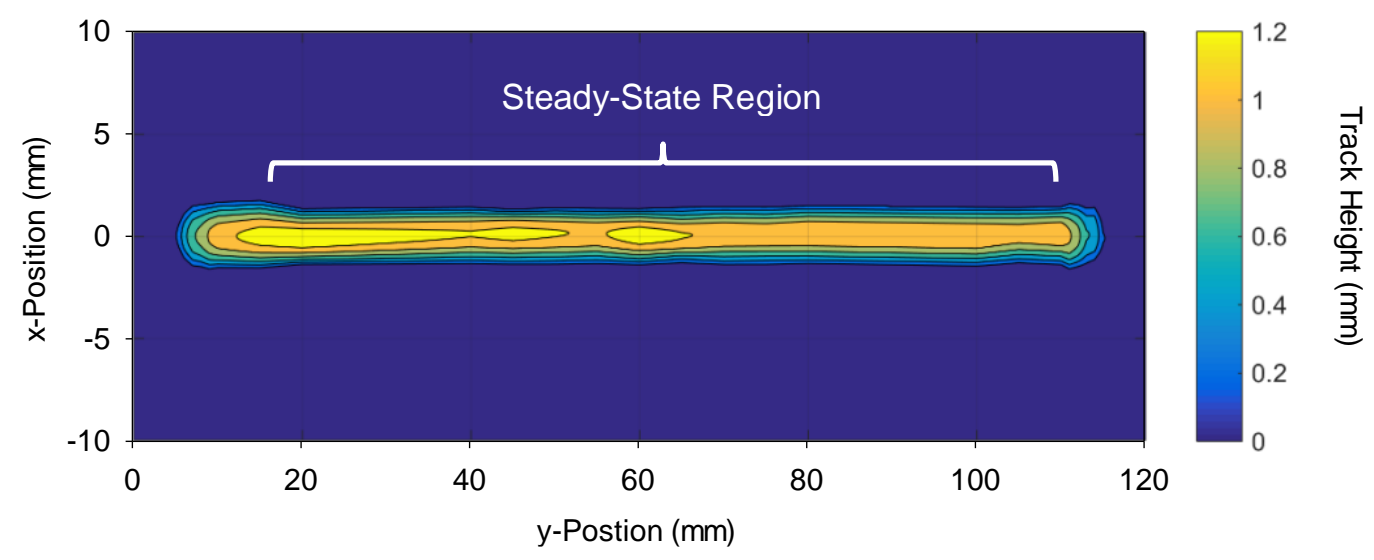

Figure 12 Contour plot showing the variation in track height derived from the surface variation microscopy data. Steady-state region displayed to show the length used to determine the average track profile.

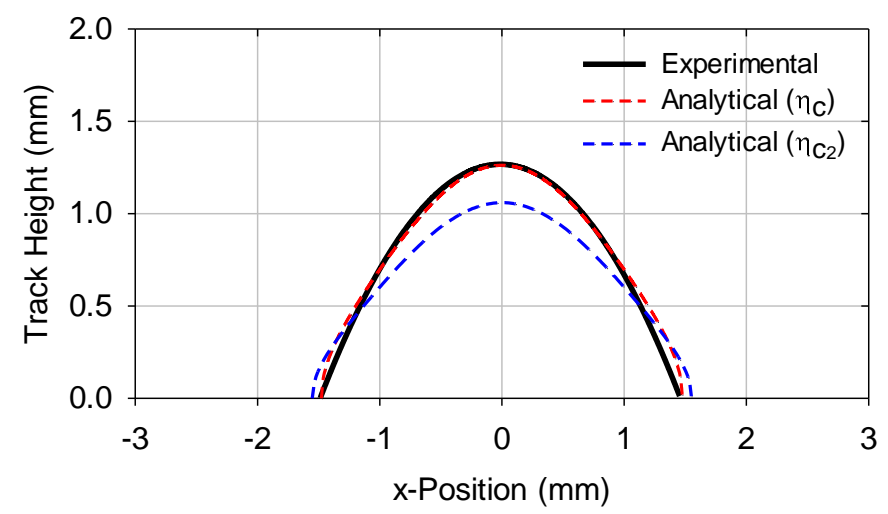

Figure 13 A comparison of the experimental and analytical track profile geometries utilising two catchment efficiency approaches, $\eta_{c}=0.51$ and $\eta_{c 2}=0.41$.

\subsection{Thermal Analysis}

Validation of the heat source was conducted through comparing in-situ temperature measurements to the predicted thermal histories. Figure 14 shows the comparison of the two data sets. Both thermal histories show a good agreement with one another, however a maximum error of $3.1 \%$ was observed at the peak temperature. The temperature gradients as the laser approaches the thermocouples during heating were predicted with good accuracy, this was also the case during the cooling phase. The predicted weld pool profile and experimental micrographs are presented in Figure 15. The isotherm from the FE model was taken at $65 \mathrm{~mm}$ in the transverse direction, at a point in time in which the fusion zone 
was at maximum width. The fusion zone is highlighted in grey, through restricting the contour to the solidus temperature $\left(1260^{\circ} \mathrm{C}\right)$. A low penetration depth of approximately 1.0 mm was observed in the micrograph. This was captured well by the FE model through utilising a small element volume directly below the track and in the weld vicinity. The overall shape of the fusion zone was predicted accurately, showing accuracy in the calibrated FE heat source model.

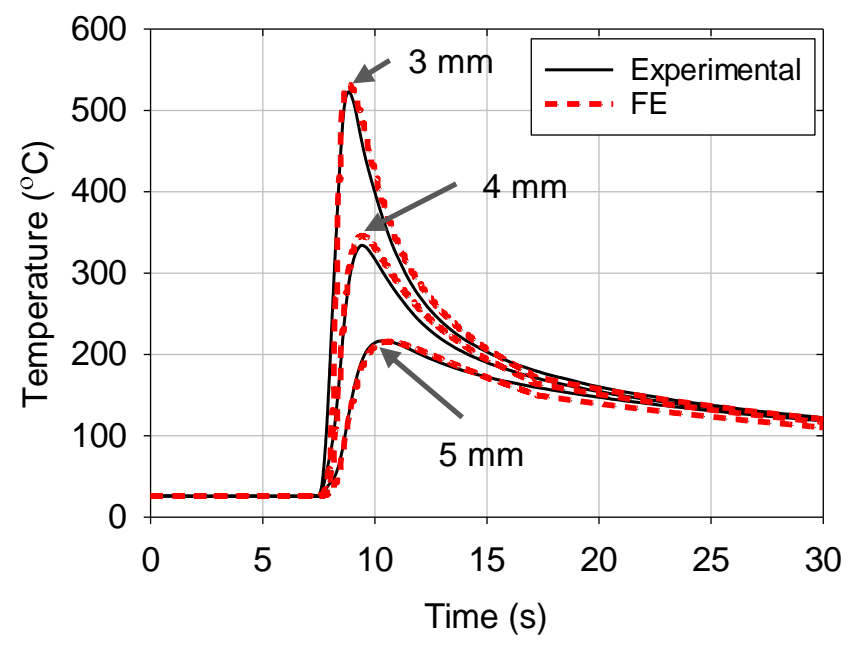

Figure 14 Comparison of experimental and numerically predicted thermal histories for three thermocouple locations positioned at the mid length of the substrate at; $3 \mathrm{~mm}, 4 \mathrm{~mm}$ and $5 \mathrm{~mm}$ from the track centreline.

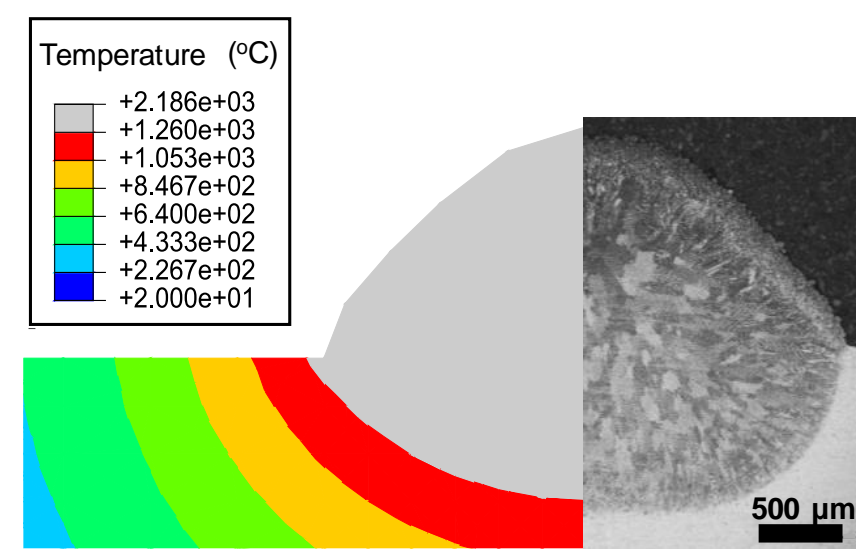

Figure 15 Comparison of the numerically predicted fusion zone and the metallographic specimen at the centre of the deposition i.e. $y=65 \mathrm{~mm}$.

\subsection{Mechanical Analysis}

To analyse the stress evolution, for the steady state region of the deposited track, all three stress components as a function of time are presented in Figure 16. The selected node was 
at the substrate mid length, on the track centre line, at the centre of the melt pool depth. Before the heat source is in the proximity of the selected node, zero stress is present throughout the substrate. The heating and cooling occurring before $t=6$ seconds has minimal effect on the stress evolution. Between the pre-heat region and the peak temperature $(\mathrm{t}=7.8$ to $t=8.95$ seconds) a balance in the direction of the stresses can be observed between all three components. As the heat source passes over the respective node, a compressive stress state exists. This arises due to the molten pool causing an expansion of the material directly below. Before and after the heat source, a tensile and compressive region develops. This is due to the cooling and subsequent contraction of material behind the heat source and the expansion of material ahead. Upon cooling, all three stress components are tensile, with the largest magnitude in the longitudinal direction. The normal stress component in this region can be assumed to equal approximately zero.

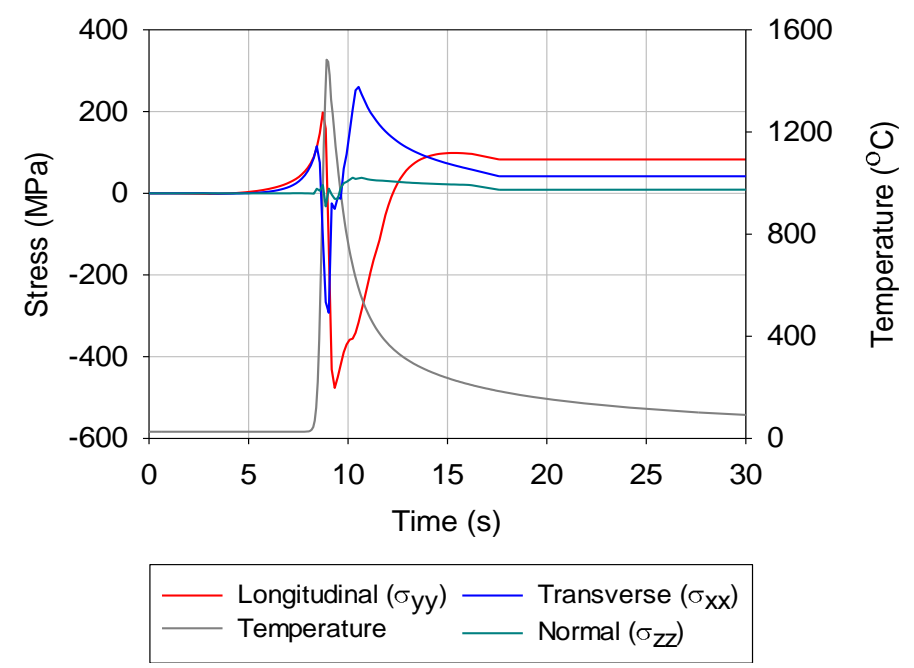

Figure 16 Numerical prediction of the transverse, longitudinal and normal stress components during the deposition process, in the steady-state track region. The nodal point selected was at the mid length of the substrate $(y=60 \mathrm{~mm})$ at the track centreline at the centre of the fusion zone $(0.375 \mathrm{~mm}$ below the substrate surface).

To coincide with Figure 16, contour plots of the through thickness stress distribution, at different time periods throughout the process, for all three stress components, are presented in Figure 17-Figure 18. Four distinct regions of the process were selected which include; the heating phase, the time at which the peak temperature was observed, the cooling phase and the final residual stress distribution at room temperature. From Figure 17, before the deposition has occurred, the stress field is predominately compressive due to the expansion of the material in front of the heat source. At the peak temperature (Figure 17b) the deposition is present which causes a tensile region to be present at the interface between the track and substrate, with the remainder of the stress field being unaltered at this stage of the process. The tensile region occurs due to the addition of track material which cools rapidly in comparison to the melt pool. Upon cooling to room temperature, the final residual 
stress state (Figure 17d) fluctuates between a compressive and tensile stress state. Figure 18a shows the longitudinal stress component during the heating phase of the process which has a similar distribution to Figure 17a, however the tensile region at the bottom surface of the substrate is not present. The stress distribution remains compressive until the cooling phase (Figure 18c) where the track and the region directly below become tensile, this was present until the specimen was cooled to room temperature. The final residual stress state for the longitudinal component in the vicinity of the Heat Affected Zone (HAZ) was tensile, this was balanced by a compressive region far field as shown in Figure 18d. For the normal stress component (Figure 19), the stress distribution does not vary discernibly during the deposition process. Below the track, during the heating phase, the stress field was compressive and for the remainder of the process the normal stress component exhibits a tensile direction. Outside the vicinity of the HAZ a small compressive region is present due to the addition of material at the substrate surface. Far field the stress distribution was tensile and upon cooling remains in this state. With regards to the stress distribution within the track, before cooling, all three components have a uniform distribution with a compressive direction being exhibited, becoming tensile during the cooling phase. Upon cooling to room temperature, a tensile region is observed between the interface of the track and substrate. This arises due to the interaction between the deposited track and the substrate. If a high stress gradient occurs in this region, separation of the track from the substrate could occur, therefore the modelling methodology presented here can also help to mitigate this issue. Averaging the FE stress predictions over the track volume presents a tensile stress state, $317.7 \mathrm{MPa}$ and $1.6 \mathrm{MPa}(24.4 \%$ and $0.1 \%$ of UTS), for the longitudinal and normal stress component respectively. A compressive stress, $9.0 \mathrm{MPa}$ ( $0.7 \%$ of UTS), in the track is observed for the transverse stress component. Therefore, it can be observed that the stress direction in the track reflects that of the substrate, directly below the track.

To validate the mechanical FE model, residual stress predictions were compared to the ND measurements. For consistency, the FE predictions were averaged over a volume equal to the gauge volume used in the experimental procedure. Figure 20 shows the comparison between the predicted and measured residual stress in the longitudinal, transverse and normal stress directions. It can be observed that the agreement between the experimentally and numerically derived residual stress profiles was good for the longitudinal and normal stress profiles. However, both the magnitude and trend for the transverse stress component differ greatly. In the vicinity of the track, the FE predictions and ND data present a tensile and compressive stress state respectively. As expected, from analysis of the stress components for each data set, at each line scan location, there is little variation present. This is because the process parameters utilised were constant throughout the deposition process. The longitudinal stress component shows the typical trend of a single pass weld at each line scan, for both the predicted and experimental values. This consists of a tensile region within the HAZ, with the peak value at the centre of the heat source. Both data sets also show a compressive region far field to balance the tensile region, with a good agreement with one 
another. For a thick substrate, the normal stress in a traditional weld has been shown to exhibit a plane stress condition [38]. However, in Figure 20 a peak stress is present at a location below the track width.
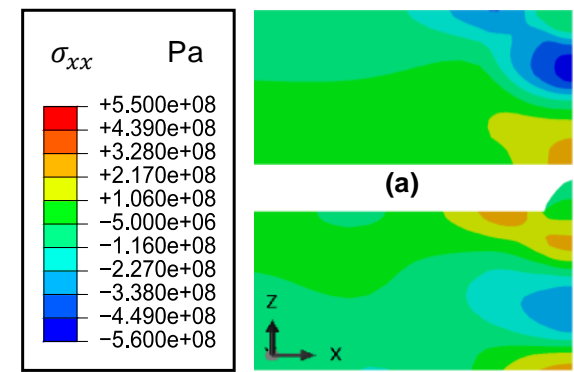

(c)

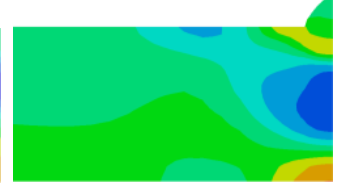

(b)

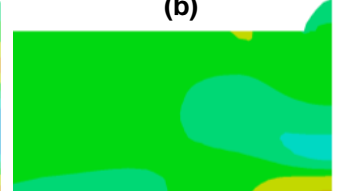

(d)

Figure 17 FE contour plots showing the through thickness stress distribution for the transverse stress component, $\sigma_{x x}$, at different time intervals through the heating and cooling phase of the process. Cross section taken at the mid length of the substrate i.e. $y=60 \mathrm{~mm}$. (a) During the heating phase at $t=8.25 \mathrm{~s}$; (b) At the peak temperature where $t=8.95 \mathrm{~s}$; (c) During the cooling phase at $\mathrm{t}=\mathbf{1 2 . 1 5} \mathrm{s}$; (d) Final residual stress field upon being fully cooled.
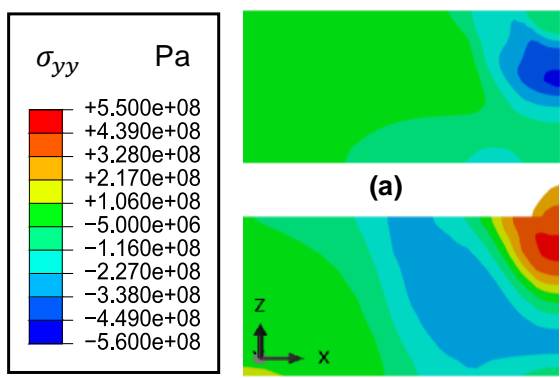

(c)

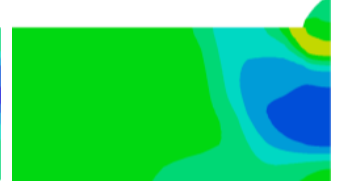

(b)

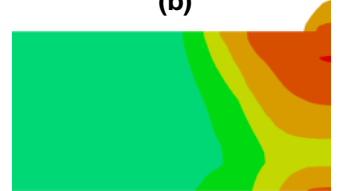

(d)

Figure 18 FE contour plots showing the through thickness stress distribution for the longitudinal stress component, $\sigma_{y y}$, at different time intervals through the heating and cooling phase of the process. Cross section taken at the mid length of the substrate i.e. $y=60 \mathrm{~mm}$. (a) During the heating phase at $t=8.25 \mathrm{~s}$; (b) At the peak temperature where $t=8.95 \mathrm{~s}$; (c) During the cooling phase at $t=12.15 \mathrm{~s}$; (d) Final residual stress field upon being fully cooled. 

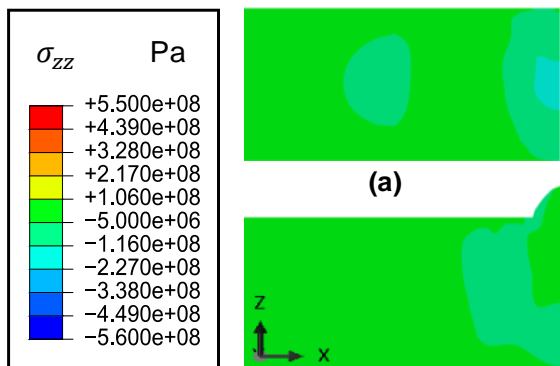

(a)

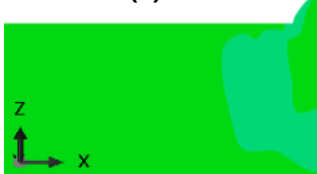

(c)

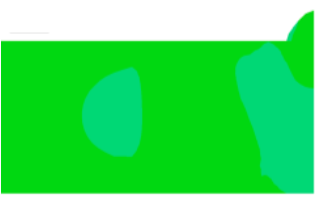

(b)

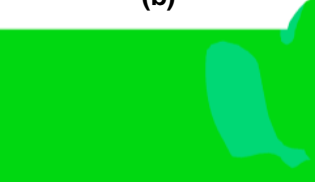

(d)

Figure $19 \mathrm{FE}$ contour plots showing the through thickness stress distribution for the normal stress component, $\sigma_{z z}$, at different time intervals through the heating and cooling phase of the process. Cross section taken at the mid length of the substrate i.e. $y=60 \mathrm{~mm}$. (a) During the heating phase at $t=8.25 \mathrm{~s}$; (b) At the peak temperature where $t=8.95 \mathrm{~s}$; (c) During the cooling phase at $\mathrm{t}=\mathbf{1 2 . 1 5} \mathrm{s}$; (d) Final residual stress field upon being fully cooled. 


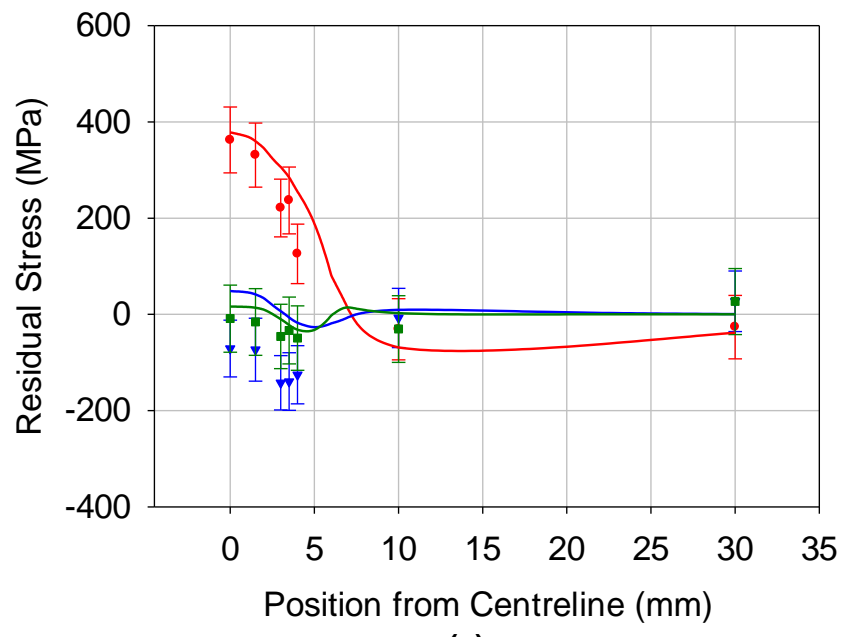

(a)

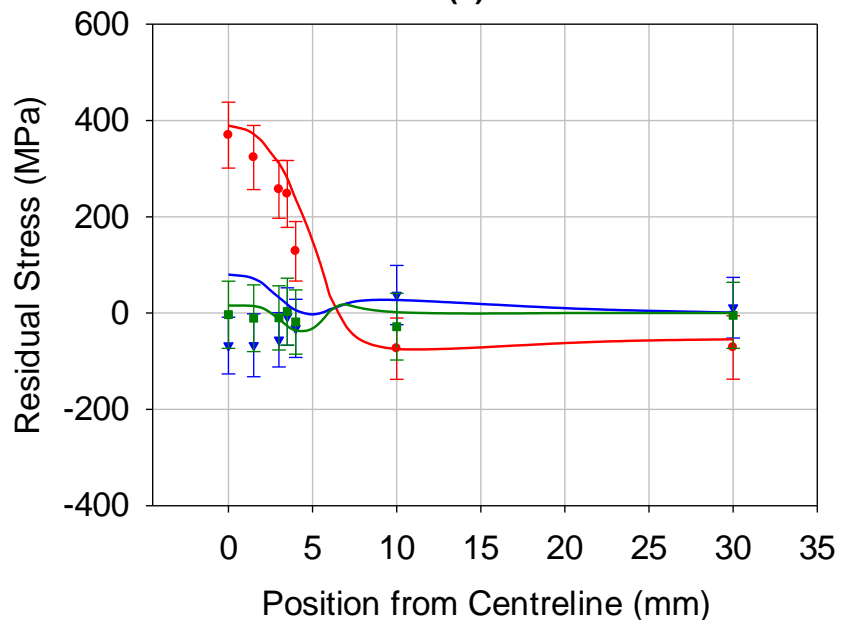

(b)

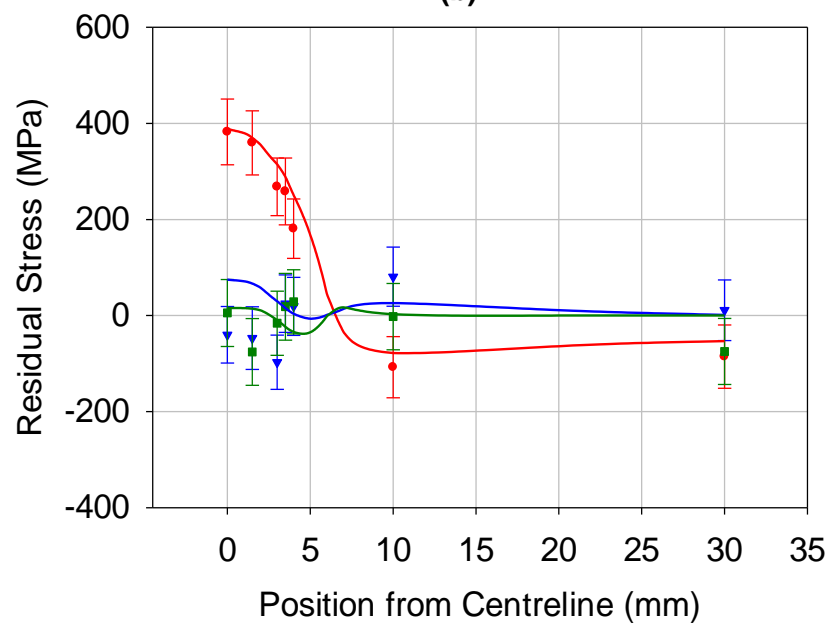

(c)

- Longitudinal ( $\left.\sigma_{y y}\right),(\mathrm{ND})$ L Longitudinal ( $\left.\sigma_{y y}\right),(\mathrm{FE})$

- Transverse $\left(\sigma_{\mathrm{XX}}\right),(\mathrm{ND})$ Transverse $\left(\sigma_{\mathrm{XX}}\right),(\mathrm{FE})$

- $\operatorname{Normal}\left(\sigma_{\mathrm{zZ}}\right),(\mathrm{ND}) \quad$ Normal $\left(\sigma_{\mathrm{zZ}}\right),(\mathrm{FE})$

Figure 20 Comparison of experimentally measured and numerically predicted tri-axial residual stresses at $2 \mathrm{~mm}$ below the surface for each line scan position; (a) y=30 mm; (b) y=60 $\mathrm{mm}$; (c) $\mathrm{y}=90 \mathrm{~mm}$. 
To further validate the model, the residual camber distortion along the length of the plate, at a position of $1.5 \mathrm{~mm}$ from the centreline was derived from the focus variation microscopy data. This was compared against the FE displacement, as shown in Figure 21. Both the FE and experimental data provide the expected parabolic trend for an unclamped substrate, with the maximum displacement at the centre of the plate. Generally, a good agreement is attained, with the maximum error equating $8 \%$ for the peak displacement.

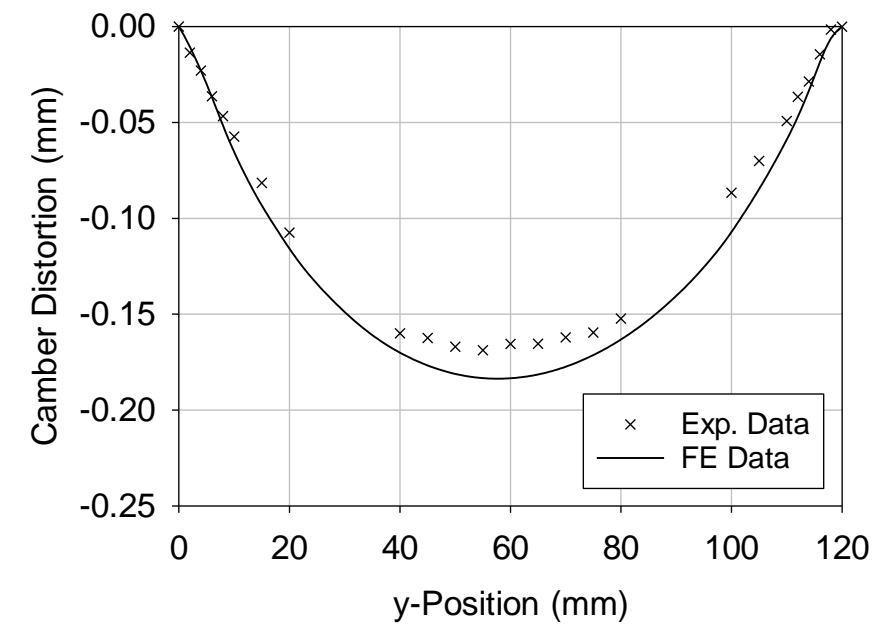

Figure 21 Comparison of experimentally measured and numerically predcited camber distortion along the substrate length.

\section{Discussion}

The work conducted in this study presents a unified modelling strategy, which encompasses the key physics and process parameters of the deposition process. The modelling strategy allows for parametric investigations to be conducted to determine the effects of the process parameters on the resultant temperature and residual stress fields; therefore, reducing the need for costly experimental trials. Through accurate predictions of the thermal and mechanical effects of the process, a tailored repair strategy can be devised and implemented.

\subsection{Track Profile and Catchment Efficiency}

Derivation of the track profile was completed analytically, utilising three approaches to define the catchment efficiency. The derived value for the unmodified catchment efficiency was 0.80 , which appears to be an overestimate when compared to the literature values which range from 0.20 - 0.65 [23], [39], [40]. When implementing the process parameters with no modification to the catchment efficiency, a converged solution could not be obtained. This is because, during the analysis the power required to sustain mass addition reduced the effective power to $239 \mathrm{~W}$. Therefore, the energy in the system could not reach an equilibrium 
state, so a stable melt pool could not be achieved. Utilising a more realistic approach defined by Partes [23] lead to a catchment efficiency of 0.41 which falls within the literature values. Implementing this value into the analytical model lead to an underestimate in the height of the derived track profile, although the width was captured well; therefore, it is apparent that the width and height of the track are predominantly governed by the melt pool geometry and the catchment efficiency respectively. The underestimate arises because the powder caught by the melt pool is accounted for twice using this approach; therefore, deriving the track profile utilising the modified catchment efficiency gives a better correlation with the experimental data; however, differences are still observed between the experimental and predicted data, more specifically in the derived width. This difference may arise from the assumption that the analytical powder flux distribution has a Gaussian profile [5], whereas in this experimental set up this may not be the case. The formulation of the analytical track height requires the height of the track to be zero at the integration limits. In reality, the nature of the process allows for a completely smooth transition between the substrate and the track material, with them both being homogenous upon cooling; therefore, the track profile would not terminate as severely at the interface between the track and substrate as observed. As the analytical model represents the process as quasi-stationary, fluid flow interaction between the molten track and substrate is neglected; therefore, no change in the geometry during solidification is accounted for. Typically, spreading of the material occurs upon cooling due to the shearing of the liquid [41], which would result in a shallow contact angle between the track and substrate. This is observed in the experimental track geometry but not in the predicted one.

\subsection{Thermal History and Melt Pool Geometry}

Numerical predictions of the thermal histories and the shape of the fusion zone correlates well with the experimental data. The heating and cooling phases agree well with the experimental data. The largest discrepancy arises at the peak temperature after the heat source passes the thermocouple. An overestimate from the FE code is to be expected, as the method in which the heat source is applied to the model is not adaptive. Therefore, the heat energy entering the mesh is not monitored or maintained [25]. The difference may also arise due to the artificial boundary condition used to model the heat sink effect between the CNC bed and the substrate. The true regime of heat loss at this location is through conduction. This may also give rise to the minimal difference observed in the cooling phase when analysing the far most thermocouple; however, to consider the CNC bed in the model would significantly increase the complexity and computational burden of the approach. It is observed that with a laser heat source, a low penetration depth is achieved in a thick substrate. This is due to a high concentration of the power flux density leading to a reduction in the size of the HAZ. It is apparent from the isotherms that the volume of elements in the vicinity of the melt pool are valid because both the shape and geometrical parameters have 
been represented well. Overall, the fusion zone of the process and the associated temperature field have been predicted with a high fidelity (3.1\% maximum error). Therefore, the expansion and contraction of the material due to the heating and cooling phases of the process would be correctly represented by the mechanical analysis.

\subsection{Residual Stress Predictions}

Validation of the mechanical FE model was conducted through the use of ND measurements. The residual stress profiles for the longitudinal and normal stress profiles correlate well. However, the trend and magnitude for the transverse component differ greatly. For all three stress components, the gradient of the stress profile far field from the weld is different. This difference may arise due to the restraining bolts changing the stress evolution. The restraints in the FE model were neglected to reduce the complexity of the simulation. Other possible reasons for the overall differences between the experimental and FE data may have arisen from; changes in temperature, microstructure changes and/or positioning errors. As the allocated beam time required measurements to be taken during the day and overnight, the measurements were not taken concurrently under identical ambient temperatures. Inconsistency in the ambient temperature will therefore reduce the validity in the derived stress values as a $1 \mathrm{~K}$ temperature rise gives rise to a change in lattice spacing by $10^{-5}$ [36], thus altering the magnitude of the stress value by $0.5 \%$. Therefore, a $10 \mathrm{~K}$ change in temperature between the strain free reference and the component being measured will give a significant effect on the lattice spacing [36]. The greatest difference between the predicted and experimental results occurred below the track. This may arise due to utilising the lattice spacing's from one strain free sample at each horizontal line scan, therefore microstructure changes and the associated intergranular stress are not accounted for [36]. Microstructure variation could occur due to different cooling rates and through varying amounts of dissolution of the powder into the melt pool at each location; therefore, the lattice parameter derived from the strain free sample may not be a true representation at the locations where the ND line scans were conducted. Positioning issues are believed to be the fundamental reason why the transverse stress profiles determined experimentally do not correlate with the FE model. It is apparent that the substrate has deformed; however, when completing the experiment, this distortion was not taken into consideration. It should also be noted that the datum surfaces chosen to use as a zero-reference location were the external faces on the $\mathrm{x}$ $y$ and y-z plane. As the surfaces were not truly square or flat, the original reference frame is not a true datum location resulting in an error being transferred to the subsequent measurement locations. This misalignment may give rise to sporadic strain values being measured due to a shift in diffraction angles as the gauge volume may have been partially filled. From further analysis of the FE model, the through thickness transverse stress profile is presented in Figure 22 for all three horizontal line scan positions. A transition in the stress state below the surface from a tensile state to compressive is observed meaning that a 
misalignment of the ND measurement location by $1 \mathrm{~mm}$ could cause a shift in the determined stress value from tensile to compressive. The trend observed in the transverse stress profile in the FE model presented in this work also agrees with the work presented by Pirch et al. [12]. It is apparent from these findings that a small misalignment of the beamline to the initial reference datum can cause a significant alteration in the stress state that is measured; therefore, the authors believe that the location in which the ND measurements were taken were at approximately $3 \mathrm{~mm}$ below the surface of the substrate. A comparison between the ND data and the FE residual stress prediction, at $3 \mathrm{~mm}$ below the surface, are presented in Figure 23. Although there are slight discrepancies between the two data sets due to the aforementioned reasons, an improved correlation is observed. Furthermore, the overall underestimate between the two data sets could be due to the plasticity model utilised (isotropic hardening). Using this plasticity model will result in an overestimation in the elastic region during cyclic loading [42] due to the underestimate in the evolution of plastic strain. This arises as no translation of the yield surface can occur, thus resulting in an underestimate of the residual stresses within the component [43]; therefore, a conservative approach should be utilised when implementing this model to predict residual stress.

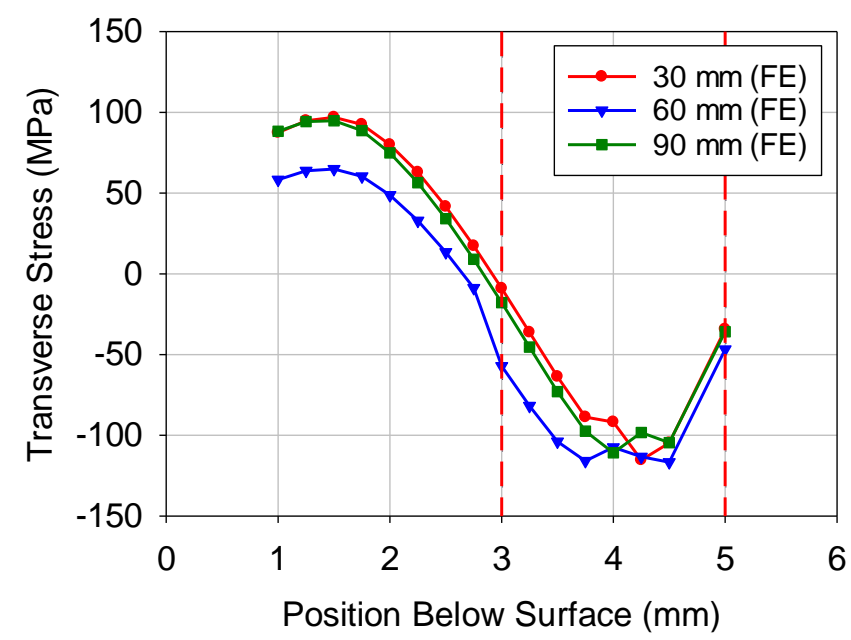

Figure 22 Residual stress prediction averaged over the ND gauge volume along the $y$-direction (transverse stress) directly below the track.

From the FE predictions and ND data, it is apparent that there are high magnitude residual stresses present. A plane stress condition is not observed through the thickness, as a peak stress at the position of the maximum track width is present. This is believed to arise due to track material contracting upon cooling, causing a compressive stress to be exerted at the substrate surface. The interaction between the track material and the substrate at this geometrical transition will give rise to the observed stress concentration. This was captured well by both the FE model and experimental data. Often, cyclic loads of varying magnitude are present in components during operation. From this study, tensile stresses are present 
throughout the substrate. The service life of the component could be severely reduced as this direction of stress promotes the initiation of material failure modes. Therefore, through heat treating or tailoring the process parameters the high magnitude residual stresses should be reduced. As the resultant stresses induced are predominately governed by thermal strain arising from the localised heating effects, an optimum power and spot size should be determined in order to control the generation of high magnitude residual stress. Cooling methods should also be investigated in an attempt to redistribute the stress, as a sharp thermal gradient is observed in the cooling phase. The modelling methods presented in this work can be used to investigate the effects of process parameters/material properties on the evolution of distortion and residual stress within the component; therefore, an ideal set of process parameters can be determined to give the required stress state after repair. 


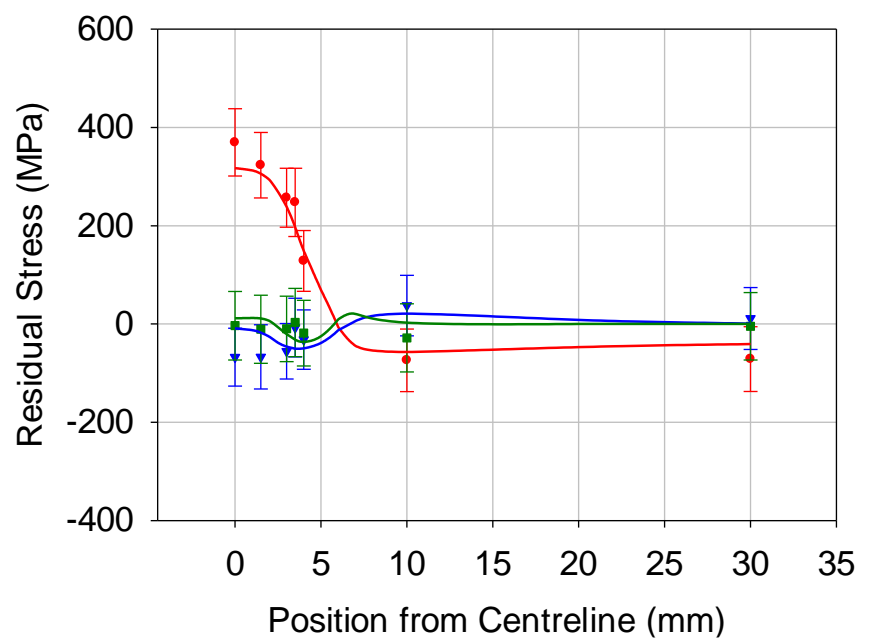

(a)

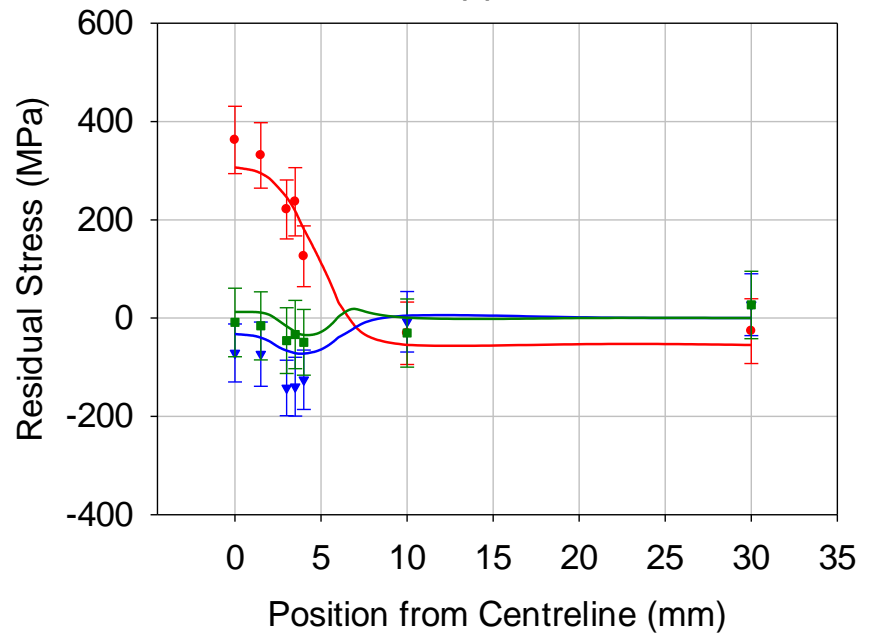

(b)

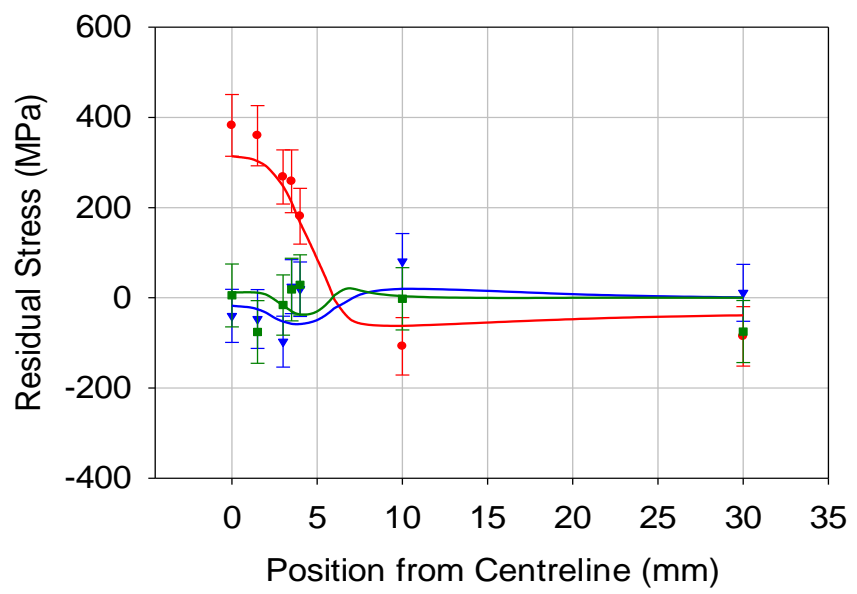

(c)

$$
\begin{array}{lll}
\text { - } & \text { Longitudinal }\left(\sigma_{\mathrm{yy}}\right),(\mathrm{ND}) & \text { Longitudinal }\left(\sigma_{\mathrm{yy}}\right),(\mathrm{FE}) \\
\text { - } & \text { Transverse }\left(\sigma_{\mathrm{xx}}\right),(\mathrm{ND}) & \text { Transverse }\left(\sigma_{\mathrm{xx}}\right),(\mathrm{FE}) \\
\text { - } & \text { Normal }\left(\sigma_{\mathrm{zz}}\right),(\mathrm{ND}) & \text { Normal }\left(\sigma_{\mathrm{zz}}\right),(\mathrm{FE})
\end{array}
$$

Figure 23 Comparison of experimentally measured and numerically predicted tri-axial residual stresses at $3 \mathrm{~mm}$ below the surface for each line scan position (a) $y=30 \mathrm{~mm}$ (b) $y=60 \mathrm{~mm}$ (c) $y=90 \mathrm{~mm}$. 


\section{Conclusions}

An improved modelling methodology has been developed to predict a realistic track geometry and to evaluate the thermomechanical effects of the DED process for a single-track 'deposition. A good agreement was attained between the experimental and predicted data sets showing confidence in the modelling methodology devised in this study. The modelling strategy can be utilised to evaluate the effect of process parameters on stress evolution, with an experimental case being presented to validate the devised modelling strategy. The following conclusions can be drawn:

- High thermal gradients and residual stresses are present, which will lead to a degradation in mechanical performance of DED parts. A method to control the evolution of high magnitude stresses for DED fabricated parts is required and can be achieved through devising optimum process parameters using the modelling strategy presented in this study. However the validity of the model is for a single track only, therefore the methodology will be extended in the future to include more complex laser scanning strategies and overlapped track geometries.

- Different methods were used to determine the catchment efficiency in this work; however, due to the importance of this parameter, further research is required to quantify this with greater accuracy to ensure that the geometry of fabricated components utilising DED can be predicted with greater precision.

- The stress profiles for a single track deposition show a similar trend to a traditional single pass weld, with the longitudinal stress component having the greatest magnitude. The transverse and normal stress components show similar trends to one another, however a plane stress condition was not exhibited in the normal direction. Validation of the stress distribution within the bead was not conducted, therefore merits further investigation.

\section{References}

[1] B. He, X. J. Tian, X. Cheng, J. Li, and H. M. Wang, "Effect of weld repair on microstructure and mechanical properties of laser additive manufactured Ti-55511 alloy," Mater. Des., vol. 119, pp. 437-445, 2017 doi:10.1016/j.matdes.2017.01.054.

[2] S. M. Thompson, L. Bian, N. Shamsaei, and A. Yadollahi, "An overview of Direct Laser Deposition for additive manufacturing; Part I: Transport phenomena, modeling and diagnostics," Addit. Manuf., vol. 8, pp. 36-62, 2015 doi:10.1016/j.addma.2015.07.001.

[3] A. J. Pinkerton, "Advances in the modeling of laser direct metal deposition," J. Laser Appl., vol. 27, no. S1, p. S15001, 2015 doi:10.2351/1.4815992.

[4] M. Picasso, C. F. Marsden, J. D. Wagniere, A. Frenk, and M. Rappaz, “A simple but 
realistic model for laser cladding," Metall. Mater. Trans. B, vol. 25, no. 2, pp. 281291, 1994 doi:10.1007/BF02665211.

[5] M. N. Ahsan and A. J. Pinkerton, "An analytical-numerical model of laser direct metal deposition track and microstructure formation," Model. Simul. Mater. Sci. Eng., vol. 19, no. 5, p. 55003, 2011 doi:10.1088/0965-0393/19/5/055003.

[6] H. E. Cline and T. R. Anthony, "Heat treating and melting material with a scanning laser or electron beam," J. Appl. Phys., vol. 48, no. 9, pp. 3895-3900, 1977 doi:10.1063/1.324261.

[7] W. Ya, B. Pathiraj, and S. Liu, "2D modelling of clad geometry and resulting thermal cycles during laser cladding," J. Mater. Process. Technol., vol. 230, pp. 217-232, 2016 doi:10.1016/j.jmatprotec.2015.11.012.

[8] N. Pirch, H. Schleifenbaum, S. Linnenbrink, A. Gasser, R. Poprawe, and M. Niessen, "Influence of process strategy on distortion and residual stresses for the powder based laser metal deposition process," Materwiss. Werksttech., vol. 48, no. 12, pp. 1304-1313, 2017 doi:10.1002/mawe.201700169.

[9] P. Michaleris, "Modeling metal deposition in heat transfer analyses of additive manufacturing processes," Finite Elem. Anal. Des., vol. 86, pp. 51-60, 2014 doi:10.1016/j.finel.2014.04.003.

[10] K. Shah, H. Khurshid, I. ul Haq, S. Anwar, and S. A. Shah, "Numerical modelling of pulsed and continuous wave direct laser deposition of Ti-6Al-4V and Inconel 718," Int. J. Adv. Manuf. Technol., vol. 95, no. 1-4, pp. 847-860, 2018 doi:10.1007/s00170017-1224-y.

[11] Y. Chew, J. H. L. Pang, G. Bi, and B. Song, "Thermo-mechanical model for simulating laser cladding induced residual stresses with single and multiple clad beads," J. Mater. Process. Technol., vol. 224, pp. 89-101, 2015 doi:10.1016/j.jmatprotec.2015.04.031.

[12] N. Pirch, M. Niessen, S. Linnenbrink, T. Schopphoven, A. Gasser, and R. Poprawe, "Temperature field and residual stress distribution for laser metal deposition," vol. 32503, pp. 1-6, 2018 doi:10.2351/1.5040634.

[13] T. Mukherjee, W. Zhang, and T. Debroy, "An improved prediction of residual stresses and distortion in additive manufacturing," Comput. Mater. Sci., vol. 126, pp. 360-372, 2017 doi:10.1016/j.commatsci.2016.10.003.

[14] X. Liang, L. Cheng, Q. Chen, Q. Yang, and A. C. To, "A modified method for estimating inherent strains from detailed process simulation for fast residual distortion prediction of single-walled structures fabricated by directed energy deposition," Addit. Manuf., vol. 23, no. August, pp. 471-486, 2018 doi:10.1016/j.addma.2018.08.029.

[15] M. Choi, R. Greif, and M. Salcudean, "A study of the heat transfer during arc welding with applications to pure metals or alloys and low or high boiling temperature materials.," Numer. Heat Transf., vol. 11, no. 4, pp. 477-489, 1987 
doi:10.1080/10407788708913566.

[16] A. J. Pinkerton and L. Li, "Modelling Powder Concentration Distribution From a Coaxial Deposition Nozzle for Laser-Based Rapid Tooling," J. Manuf. Sci. Eng., vol. 126, no. 1, pp. 33-41, 2004 doi:10.1115/1.1643748.

[17] A. J. Pinkerton, R. Moat, K. Shah, L. Li, M. Preuss, and P. J. Withers, "A Verified Model of Laser Direct Metal Deposition Using an Analytical Enthalpy Balance Method," Proc. 26th Int. Congr. Appl. Lasers Electro-optics, 2007.

[18] K. Dai and L. Shaw, "Thermal and stress modeling of multi-material laser processing," Acta Mater., vol. 49, no. 20, pp. 4171-4181, 2001 doi:10.1016/S13596454(01)00312-3.

[19] D. Dye, O. Hunziker, S. M. Roberts, and R. C. Reed, "Modeling of the mechanical effects induced by the tungsten inert-gas welding of the IN718 superalloy," Metall. Mater. Trans. A, vol. 32, no. 7, pp. 1713-1725, 2001 doi:10.1007/s11661-001-0149$\mathrm{z}$.

[20] J. J. Valencia and Quested P.N, "Thermophysical Properties," ASM Handb. Cast., vol. 15, no. Ref 24, pp. 468-481, 2008 doi:10.1361/asmhba0005240.

[21] C. L. Chan and J. Mazumder, "One-dimensional steady-state model for damage by vaporization and liquid expulsion due to laser-material interaction," J. Appl. Phys., vol. 62, no. 11, pp. 4579-4586, 1987 doi:10.1063/1.339053.

[22] M. Anderson, R. Patwa, and Y. C. S. Ã, "Laser-assisted machining of Inconel 718 with an economic analysis," vol. 46, pp. 1879-1891, 2006 doi:https://doi.org/10.1016/j.ijmachtools.2005.11.005.

[23] K. Partes, "Analytical model of the catchment efficiency in high speed laser cladding," Surf. Coatings Technol., vol. 204, no. 3, pp. 366-371, 2009 doi:10.1016/j.surfcoat.2009.07.041.

[24] L. E. Lindgren, A. Lundback, M. Fisk, R. Pederson, and J. Andersson, "Simulation of additive manufacturing using coupled constitutive and microstructure models," Addit. Manuf., vol. 12, pp. 144-158, 2016 doi:10.1016/j.addma.2016.05.005.

[25] A. A. Deshpande, D. W. J. Tanner, W. Sun, T. H. Hyde, and G. McCartney, "Combined butt joint welding and post weld heat treatment simulation using SYSWELD and ABAQUS," Proc. Inst. Mech. Eng. Part L J. Mater. Des. Appl., vol. 225, no. 1, pp. 1-10, 2011 doi:10.1177/14644207JMDA349.

[26] M. N. A. and A. J. Pinkerton, "An analytical-numerical model of laser direct metal deposition track and microstructure formation," Model. Simul. Mater. Sci. Eng., vol. 19, no. 5, p. 55003, 2011 doi:10.1088/0965-0393/19/5/055003.

[27] W. Perret, "Welding Simulation of Complex Automotive Welded Assembly Possibilities and Limits of the Application of Analytical Temperature Field Solutions," Tech- nische Universität Berlin, Fakultät V - Verkehrs - und Maschinensysteme, 2013.

[28] R. W. Lewis, K. Morgan, H. R. Thomas, and K. Seetharamu, The Finite Element 
Method in Heat Transfer Analysis. John Wiley \& Sons, 1996.

[29] D. Deng and H. Murakawa, "Numerical simulation of temperature field and residual stress in multi-pass welds in stainless steel pipe and comparison with experimental measurements," Comput. Mater. Sci., vol. 37, no. 3, pp. 269-277, 2006 doi:10.1016/j.commatsci.2005.07.007.

[30] V. Pavelic, R. Tanbakuchi, O. . Uyehara, and P. . Myers, "Welding Journal Research Supplement," vol. 48, pp. 295-305, 1969.

[31] P. Foteinopoulos, A. Papacharalampopoulos, and P. Stavropoulos, "On thermal modeling of Additive Manufacturing processes," CIRP J. Manuf. Sci. Technol., vol. 20, pp. 66-83, 2018 doi:10.1016/j.cirpj.2017.09.007.

[32] H. Yoshimura and K. Winterton, "Solidification Mode of Weld Metal in Inconel 718," Weld. J. Res. Suppl., vol. 51, pp. 132-137, 1972.

[33] ABAQUS Analysis User's Manual Version 6.13. USA: Dassault Systèmes Simulia Corp, 2013.

[34] T. E. Abioye, J. Folkes, and A. T. Clare, "A parametric study of Inconel 625 wire laser deposition," J. Mater. Process. Technol., vol. 213, no. 12, pp. 2145-2151, 2013 doi:10.1016/j.jmatprotec.2013.06.007.

[35] J. R. Santisteban, M. R. Daymond, J. A. James, and L. Edwards, "ENGIN-X: A thirdgeneration neutron strain scanner," J. Appl. Crystallogr., vol. 39, no. 6, pp. 812-825, 2006 doi:10.1107/S0021889806042245.

[36] P. J. Withers, M. Preuss, A. Steuwer, and J. W. L. Pang, "Methods for obtaining the strain-free lattice parameter when using diffraction to determine residual stress," $J$. Appl. Crystallogr., vol. 40, no. 5, pp. 891-904, 2007 doi:10.1107/S0021889807030269.

[37] D. Dye, S. M. Roberts, P. J. Withers, and R. C. Reed, "The determination of the residual strains and stresses in a tungsten inert gas welded sheet of IN718 superalloy using neutron diffraction," J. Strain Anal. Eng. Des., vol. 35, no. 4, pp. 247259, 2000 doi:10.1243/0309324001514396.

[38] G. Salerno et al., "On the interaction between welding residual stresses: a numerical and experimental investigation $0 n$ the interaction between welding residual stresses: a numerical and experimental investigation," Int. J. Mech. Sci., vol. 144, no. 17, pp. 654-667, 2018 doi:10.1016/j.jimecsci.2018.04.055.

[39] J. Lin, "Laser attenuation of the focused powder streams in coaxial laser cladding," vol. 28, no. 2000, pp. 27-33, 2015 doi:10.2351/1.521910.

[40] Y. Huang, M. B. Khamesee, and E. Toyserkani, "A comprehensive analytical model for laser powder-fed additive manufacturing," Addit. Manuf., vol. 12, pp. 90-99, 2016 doi:10.1016/j.addma.2016.07.001.

[41] H. Zhang, "Theoretical analysis of spreading and solidification of molten droplet during thermal spray deposition," Int. J. Heat Mass Transf., vol. 42, no. 14, pp. 24992508, 1999 doi:10.1016/S0017-9310(98)00364-0. 
[42] O. Muránsky, C. J. Hamelin, M. C. Smith, P. J. Bendeich, and L. Edwards, "The effect of plasticity theory on predicted residual stress fields in numerical weld analyses,"

Comput. Mater. Sci., vol. 54, no. 1, pp. 125-134, 2012

doi:10.1016/j.commatsci.2011.10.026.

[43] O. Muránsky, C. J. Hamelin, V. I. Patel, V. Luzin, and C. Braham, "The influence of constitutive material models on accumulated plastic strain in finite element weld analyses," Int. J. Solids Struct., vol. 69-70, pp. 518-530, 2015

doi:10.1016/j.ijsolstr.2015.04.032. 